\title{
Microsurgical anatomy of the central core of the brain
}

\author{
Eduardo Carvalhal Ribas, MD, PhD, ${ }^{1-3}$ Kaan Yağmurlu, MD, ${ }^{1}$ Evandro de Oliveira, MD, $\mathrm{PhD},{ }^{4,5}$ \\ Guilherme Carvalhal Ribas, MD, PhD, ${ }^{3,6}$ and Albert Rhoton Jr., MD, PhD ${ }^{1}$
}

1Department of Neurosurgery, University of Florida, Gainesville, Florida; ${ }^{2 D i v i s i o n ~ o f ~ N e u r o s u r g e r y, ~ H o s p i t a l ~ d a s ~ C l i ́ n i c a s, ~ a n d ~}$ ${ }^{6}$ Department of Surgery, University of São Paulo Medical School; ${ }^{3} \mathrm{Hospital}$ Israelita Albert Einstein; and ${ }^{5}$ Institute of Neurological Sciences, São Paulo, São Paulo, Brazil; and `Department of Neurological Surgery, Mayo Clinic, Jacksonville, Florida

\begin{abstract}
OBJECTIVE The purpose of this study was to describe in detail the cortical and subcortical anatomy of the central core of the brain, defining its limits, with particular attention to the topography and relationships of the thalamus, basal ganglia, and related white matter pathways and vessels.
\end{abstract}

METHODS The authors studied 19 cerebral hemispheres. The vascular systems of all of the specimens were injected with colored silicone, and the specimens were then frozen for at least 1 month to facilitate identification of individual fiber tracts. The dissections were performed in a stepwise manner, locating each gray matter nucleus and white matter pathway at different depths inside the central core. The course of fiber pathways was also noted in relation to the insular limiting sulci.

RESULTS The insular surface is the most superficial aspect of the central core and is divided by a central sulcus into an anterior portion, usually containing 3 short gyri, and a posterior portion, with 2 long gyri. It is bounded by the anterior limiting sulcus, the superior limiting sulcus, and the inferior limiting sulcus. The extreme capsule is directly underneath the insular surface and is composed of short association fibers that extend toward all the opercula. The claustrum lies deep to the extreme capsule, and the external capsule is found medial to it. Three fiber pathways contribute to form both the extreme and external capsules, and they lie in a sequential anteroposterior disposition: the uncinate fascicle, the inferior fronto-occipital fascicle, and claustrocortical fibers. The putamen and the globus pallidus are between the external capsule, laterally, and the internal capsule, medially. The internal capsule is present medial to almost all insular limiting sulci and most of the insular surface, but not to their most anteroinferior portions. This anteroinferior portion of the central core has a more complex anatomy and is distinguished in this paper as the "anterior perforated substance region." The caudate nucleus and thalamus lie medial to the internal capsule, as the most medial structures of the central core. While the anterior half of the central core is related to the head of the caudate nucleus, the posterior half is related to the thalamus, and hence to each associated portion of the internal capsule between these structures and the insular surface. The central core stands on top of the brainstem. The brainstem and central core are connected by several white matter pathways and are not separated from each other by any natural division. The authors propose a subdivision of the central core into quadrants and describe each in detail. The functional importance of each structure is highlighted, and surgical approaches are suggested for each quadrant of the central core.

CONCLUSIONS As a general rule, the internal capsule and its vascularization should be seen as a parasagittal barrier with great functional importance. This is of particular importance in choosing surgical approaches within this region.

https://thejns.org/doi/abs/10.3171/2017.5.JNS162897

KEY WORDS central core; insula; anterior commissure; claustrocortical fibers; internal capsule; inferior fronto-occipital fascicle; uncinate fascicle; anatomy; neuroanatomy

$\mathrm{T}$ HE human brain has a complex anatomy, and to favor its understanding, to organize its nomenclature, and for clinical practice, it has been arbitrarily divided into lobes, regions, and compartments. Since these divisions correspond to different concepts, they have been created according to different criteria, and hence they can overlap but are definitely complementary to each other and can be helpful in different ways. The concept of a central core within each cerebral hemisphere stands out as a natural and obvious anatomical delimitation with important clinical and surgical implications.

The central core stands deep inside each hemisphere of

ABBREVIATIONS AIP = anterior insular point; ALS = anterior limiting sulcus; $A P S=$ anterior perforated substance; FLP = frontal limen point; IFOF = inferior fronto-occipital fascicle; ILS = inferior limiting sulcus; LGB = lateral geniculate body; PIP = posterior insular point; SLS = superior limiting sulcus; TLP = temporal limen point; UF = uncinate fascicle.

SUBMITTED November 20, 2016. ACCEPTED May 15, 2017.

INCLUDE WHEN CITING Published online December 22, 2017; DOI: 10.3171/2017.5.JNS162897. 
the brain, as a solid and well-delimited block between the brainstem and the cerebral lobes. It is basically composed of the insular surface, basal ganglia, and thalamus, connected to the rest of the supratentorial compartment by the cerebral isthmus, represented by the continuation of the internal, external, and extreme capsules toward the cerebral lobes. It also harbors part of the anterior commissure, part of the amygdalofugal pathways, and the substantia innominata region. Being at the morphological center of the brain, the central core serves to integrate information, and it has an important role in motor and sensory functions, emotion, and cognition. ${ }^{4}$

From a neurosurgical standpoint, the central core concept of the brain is particularly justified since the complexity and possible morbidities of surgery in this region are very relevant. Even though a few authors have already described the central core as a distinct region, ${ }^{4,30,35}$ the current study was undertaken to better define its limits, describe its contents, and clarify the complex relation between its structures. We also propose a subdivision of the central core into quadrants with the aim of making operations involving this region safer and more precise.

\section{Methods}

The cortical and subcortical anatomy of the central core and its relationship to the rest of the brain were studied in 19 adult cerebral hemispheres, at the University of Florida, with the aid of $\times 3$ to $\times 40$ microscope magnification. The specimens were first prepared with formalin, and then preserved in $70 \%$ alcohol. The vessels were perfused with colored latex, and fiber dissection was performed after the specimens had been frozen for at least 1 month.

Freezing the brain leads to water expansion and the formation of ice crystals, resulting in the spreading of white matter fibers and facilitating the individualization of these structures during dissection. Although the smallest individual features of each fiber cannot be seen due to the dense subcortical fiber network and because the dissection and exposure of each fiber tract often results in the destruction of other fiber tracts, the main pathways can be identified, allowing a better understanding of the subcortical connections of the brain. This freezing technique, first described by Joseph Klingler ${ }^{19}$ and later revitalized by Türe et al., ${ }^{41}$ has already been reported to have a good correlation with diffusion tensor imaging (DTI) tractography. ${ }^{24}$

The dissection was carried out in a stepwise manner, from lateral to medial and from medial to lateral. The insular surface is the most lateral aspect of the central core, and it is exposed after opening of the sylvian fissure and retraction of its opercula. After the superficial characteristics were noted, the insula was first "decorticated" with the aid of dissectors, and as the dissection progressed deeper, the white matter pathways and deep structures inside this region were revealed. These pathways are organized in different planes, and the deep structures occupy spaces between them. The location of each fiber pathway and deep structure was examined, with special attention to their position under the limiting sulci of the insula.

The specimens were transfixed from medial to lateral by needles placed perpendicular to the medial cerebral surface at the following specific landmarks to identify the location of their lateral projection over the insular surface: foramen of Monro, medial aspect of anterior commissure, floor of the anterior horn of the lateral ventricle, lateral geniculate body (LGB) of the thalamus, anterior limit of the cerebral peduncle, and superior-most aspect of the thalamus. Together with the location of the anterior-most aspect of the precentral gyrus, these data were used to subdivide the central core into quadrants in an attempt to promote an easier understanding of this region's anatomy.

All measurements were made independently by the first and the second authors (E.C.R. and K.Y.), using an electronic digital caliper, and the values presented here are the average of both findings. Statistical analysis was performed later using IBM SPSS software (IBM Corp). Digital single-lens reflex (SLR) cameras (Nikon and Canon) were used for photographic documentation, and the pictures were processed by the high-dynamic-range imaging (HDRI or HDR) method using the software Photomatix Pro (HDRsoft). This method can combine several pictures taken from the same point of view, but with different exposure values, enhancing the contrast of the final picture to better display the white matter pathways.

\section{Results \\ Defining the Central Core}

The central core stands as a block on top of the brainstem, at the morphological center of the supratentorial compartment. This solid block includes, from lateral to medial, the insular surface, the extreme capsule, the claustrum, the external capsule, the putamen, the globus pallidus, the internal capsule, the caudate nucleus, the stria terminalis, the septal region, and the thalamus. It also includes the medial part of the anterior commissure and the structures located above and posterior to the anterior perforated substance (i.e., the nucleus accumbens, which corresponds to the ventral striatum; the Meynert nucleus; the ventral amygdalofugal pathways; and the substantia innominata, which roughly corresponds to the ventral globus pallidus). Superiorly, it is connected to the rest of the brain by the cerebral isthmus, and inferiorly by fibers that project from and toward the brainstem (Fig. 1).

In the cerebral isthmus, all fibers arising from the central core and radiating to all other cerebral lobes are seen together as a group. It is composed of the continuation of the extreme, external, and internal capsules; the lateral extension of the anterior commissure; and the ventral amygdalofugal pathways. All of these white matter fibers are located inside the central core but spread toward the convexity of the brain. This isthmus becomes more conspicuous under and around the limiting sulci of the insula, where these fibers pass through the narrow space between the limiting sulci and the lateral ventricle cavity and become close enough to each other to assume the shape of a single and thick bundle of fibers.

The insular surface is the most lateral part of the central core and is observed by splitting the sylvian fissure and retracting the frontoparietotemporal opercula (Fig. 1). It is limited by the anterior, superior, and posterior 

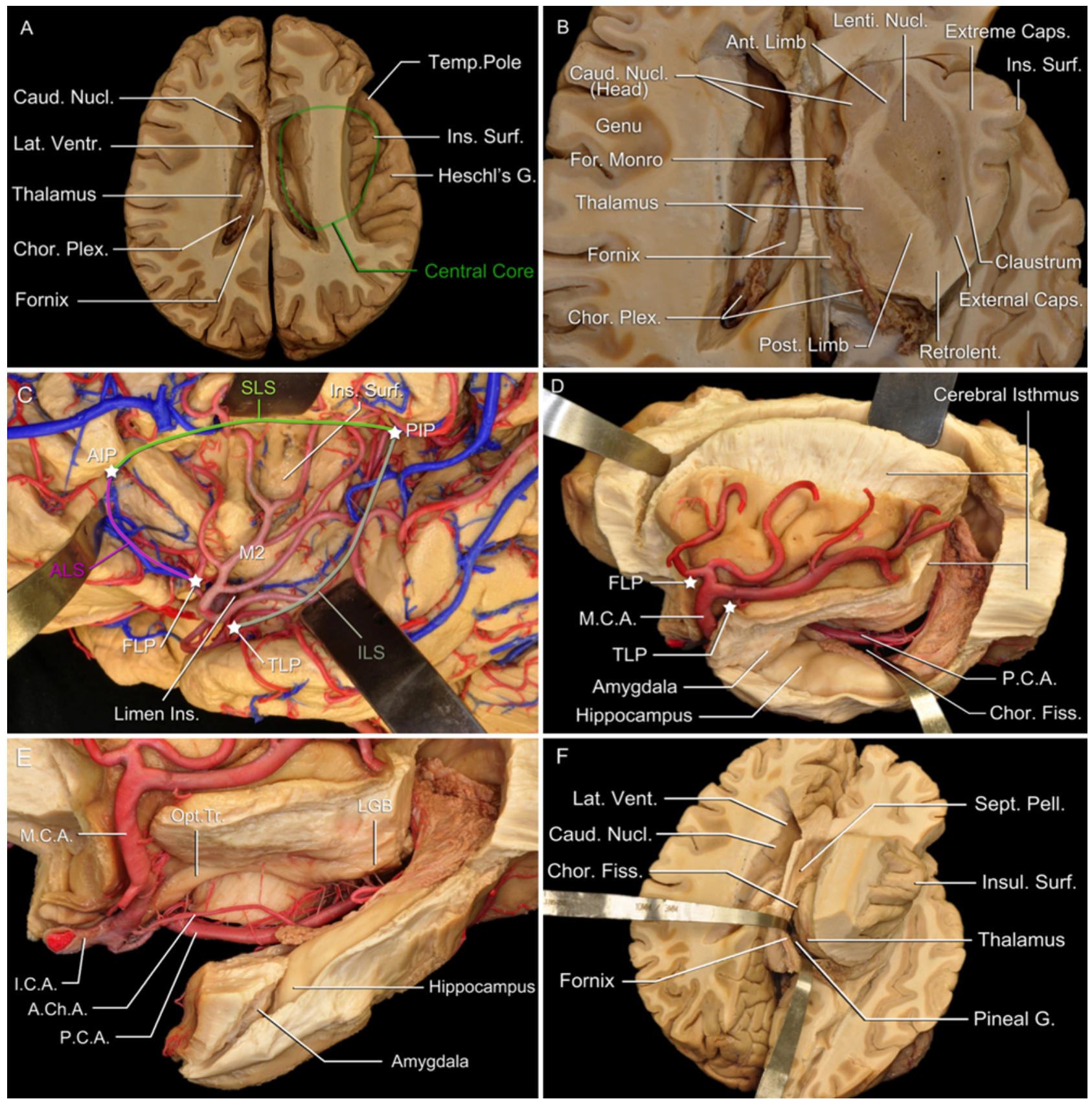

FIG. 1. The central core at the center of the brain. A: An axial cut of the brain at the level of the middle frontal gyrus was made, and the frontal operculum over the insular surface was removed. The central core is exposed and found at the morphological center of the brain, with its medial aspects represented by the caudate nucleus and the thalamus found and with its lateral aspect represented by the insular surface. B: An axial cut was performed through the central core, revealing its composition by basal ganglia and fiber pathways. These fiber pathways are organized inside the central core into capsules and named according to their position in relation to the basal ganglia: the extreme capsule is found between the insular surface and the claustrum, the external capsule between the claustrum and the lentiform nucleus, and the internal capsule (formed by the anterior limb, genu, posterior limb, and retrolenticular and sublenticular portions) between the lentiform nucleus laterally and the caudate nucleus and thalamus medially. C: The insular surface is the most superficial aspect of the central core, has a triangular shape, and is easily seen after the sylvian fissure is split. The insular surface is bounded by the anterior, superior, and inferior limiting sulci, with the limen insula at its anteroinferior vertex. The meeting points of these sulci were used in this study and were defined as follows: anterior insular point (where the anterior and superior limiting sulci meet), posterior insular point (where the superior and inferior limiting sulci meet), frontal limen point (where the limen and the anterior limiting sulcus meet), and temporal limen point (where the limen and the inferior limiting sulcus meet). D: A deep cut made around the insular surface, at the limiting sulci, passes through the cerebral isthmus, reaching the lateral ventricle and detaching the central core from the rest of the cerebral hemisphere. FIG. 1. (continued) $\rightarrow$ 
FIG. 1. E: Most temporal mesial structures are already separated from the central core by the choroidal fissure, a natural cleft between the fornix and the thalamus. The temporal pole and the amygdala are attached to the central core and can be separated by a line traced from the bifurcation of the internal carotid artery or the proximal segment of $M_{1}$ to the inferior choroidal point (carotidchoroidal line), just lateral to the optic tract. F: The frontal, parietal, and occipital lobes were removed, and the choroidal fissure is seen inside the lateral ventricle. This circular cleft naturally separates the thalamus from the hippocampal formation (fornix and hippocampus), and its opening will reach the roof of the third ventricle (velum interpositum cistern), the pineal region (quadrigeminal cistern), and the parapeduncular region (ambient cistern). A.Ch.A. = anterior choroidal artery; AIP = anterior insular point; ALS = anterior limiting sulcus; Ant. Limb = anterior limb; Caps. = capsule; Caud. Nucl. = caudate nucleus; Chor. Fiss. = choroidal fissure; Chor. Plex. = choroid plexus; FLP = frontal limen point; For. Monro = foramen of Monro; G. = gland; Heschl's G. = Heschl's gyrus; I.C.A. = internal carotid artery; ILS = inferior limiting sulcus; Ins. = insula; Insul. Surf. = insular surface; Lat. Ventr. = lateral ventricle; Lenti. Nucl. = lentiform nucleus; LGB = lateral geniculate body; M.C.A. = middle cerebral artery; Opt. Tr. = optic tract; P.C.A. = posterior cerebral artery; PIP = posterior insular point; Post. Limb = posterior limb; Retrolent. = retrolenticular; Sept. Pell. = septum pellucidum; SLS = superior limiting sulcus; Temp. = temporal; TLP = temporal limen point; Vent. = ventricle. Anatomical dissections performed by Eduardo Carvalhal Ribas, MD, at Dr. Rhoton's laboratory. Reproduced with permission from the Rhoton Collection (http://rhoton.ineurodb.org), CC BY-NC-SA 4.0 (http://creativecommons.org/licenses/by-nc-sa/4.0).

insular limiting sulci (also referred to all together as the circular sulcus of the insula). ${ }^{38,39}$ The limits of the central core were first defined by 3 planes, each drawn from one of the limiting sulci toward the lateral ventricle, marking the anterior, superior, and posteroinferior limits. Each of these planes passes through the cerebral isthmus, transecting the connections that link the central core to the other cerebral lobes.

The anterior plane is directed medially and connects the anterior limiting sulcus (ALS) to the anterior horn of the lateral ventricle, just anterior to the head of the caudate nucleus. This plane marks the anterior limit of the central core, leaving the genu of the corpus callosum excluded anteriorly, and all of the caudate nucleus included posteriorly. Only the superior part the ALS is related medially to the lateral ventricle because, as this plane continues inferiorly until the beginning of the ALS at the limen insula, it passes in front of the septal region. As a result, the septal region was also included as part of the central core concept. It is composed of 3 small gyri: the anterior and posterior paraolfactory gyri and the paraterminal gyrus, separated by the anterior and posterior paraolfactory sulci.

The superior plane is also directed medially and connects the superior limiting sulcus (SLS) to the body and superior aspect of the atrium of the lateral ventricle, just superior to the body of the caudate nucleus. The superior margin of the caudate nucleus is slightly more superior than the SLS, making this superior plane slightly oblique. This plane marks the superior limit of the central core, with the corpus callosum excluded superiorly and the caudate nucleus included inferiorly. The inferior plane is directed medially at its posterior part and inferiorly at its inferior part, connecting the inferior limiting sulcus (ILS) to the atrium and to the inferior horn of the lateral ventricle, respectively.

As these 3 planes reach the lateral ventricle, they are later connected to the choroidal fissure. This fissure is a natural cleft between the thalamus and the fornix in the lateral ventricle, extending in a $\mathrm{C}$-shaped arc from the foramen of Monro and coursing around the superior, posterior, and inferior surfaces of the thalamus to its inferior termination, called the inferior choroidal point, which is located just behind the head of the hippocampus. Use of this fissure excludes most limbic structures (the parahippocampal gyrus, amygdala, hippocampus, and fornix) from the central core concept, but they have an intimate relation with it as they encircle this region, and the caudate nucleus and stria terminalis (running in the groove between the thalamus and caudate nucleus) are included inside the central core.

The anterior plane first reaches the midline just in front of the septal region and then turns posteriorly toward the foramen of Monro to enter the third ventricle. This course is lateral to the columns of the fornix, leaving this structure excluded from the central core at the midline. The superior plane passes through the choroidal fissure and the velum interpositum and reaches the roof of the third ventricle. The inferior plane, as it passes through the choroidal fissure, gains access to the quadrigeminal cistern in its posterior aspect and to the ambient cistern more inferiorly. Finally, a straight line connects the inferior choroidal point to the limen insula, detaching the amygdala and the temporal pole from the central core. As a result, cuts made at the anterior, superior, and inferior planes will detach the central core from the frontal, parietal, and temporal lobes, respectively.

These limits depict the central core encircled outside by the lateral ventricles and important structures of the limbic system (fornix and parahippocampal gyrus), together approximating a C-shaped morphology that embraces its superior, posterior, and lateroinferior borders (Fig. 1). The third ventricle, hypothalamus, and epithalamus are grouped together in the midline, between the central cores of the left and right hemispheres.

The medial-inferior limit of the central core is represented by the hypothalamic sulcus, a shallow sulcus that runs from the foramen of Monro toward the opening of the aqueduct of Sylvius and separates the thalamus dorsally from the hypothalamus ventrally. The medial-posterior limit of the central core is represented by the groove between the thalamus and the epithalamus, which consists of the pineal body, the right and left habenular nuclei, and the habenular and posterior commissures. Both the hypothalamus and the epithalamus are singular regions and are best understood as being separated from the central core, which is present in both hemispheres.

The anteroinferior limit of the central core is not well defined, as this region continues into the orbitofrontal cortex and cingulate gyrus without a natural cleft or fissure between them. The anterior limit of the anterior perforat- 
TABLE 1. Cortical analysis of all specimens: insular surface aspects and length of the insular limiting sulci

\begin{tabular}{|c|c|}
\hline Feature & Findings \\
\hline No. of short gyri & 3 in 11 specimens (58\%), 4 in 7 specimens (37\%), 2 in 1 specimen $(5 \%)$ \\
\hline No. of long gyri & 2 in 11 specimens (95\%), 1 in 1 specimen $(5 \%)$ \\
\hline Description of central insular sulcus & Continuous in 11 specimens (95\%), interrupted in 1 specimen (5\%) \\
\hline Insular apex description & Well defined in 13 specimens (68\%), not well defined in 6 specimens $(32 \%)$ \\
\hline Transverse gyrus presence & Present in all specimens $(100 \%)$ \\
\hline Accessory gyrus presence & Present in 8 specimens (50\%), absent in 8 specimens (50\%) \\
\hline \multicolumn{2}{|l|}{ Length of limiting sulci in $\mathrm{mm}^{*}$} \\
\hline ALS & $26.29 \pm 4.29(16.88-35.50)$ \\
\hline SLS & $51.74 \pm 6.50(35.02-65.67)$ \\
\hline ILS & $44.03 \pm 4.52(33.06-50.44)$ \\
\hline
\end{tabular}

* Mean \pm SD (range).

ed substance (APS), defined as the medial and lateral olfactory striae, is connected to the anterior limit plane, also including this region as the bottom part of the central core. The orbitofrontal cortex, cingulate gyrus, and genu of the corpus callosum are excluded anteriorly, not being understood as part of the central core. The structures above the APS were grouped into the "anterior perforated substance region" (APS region) and are described in detail later.

There is also no clear division between the midbrain and central core, but we considered this transition as a line drawn inferior to the optic tract and the lateral geniculate body (LGB). The thalamus is the most inferior structure of the central core, with the medial and lateral geniculate bodies being its most inferolateral substructures.

\section{Insular Surface}

The insular surface is the most lateral aspect of the central core and is encircled by the insular limiting sulci. The limen insula is a hook-like structure found at the anteroinferior vertex of the insula and is connected to the anterior and inferior limiting sulci (ALS and ILS). The point where the limen joins the ALS, at the frontal lobe, we refer to as the "frontal limen point" (FLP) and consider the beginning of the ALS. This sulcus ends at its meeting point with the SLS, which is referred to as the anterior insular point (AIP) ${ }^{39}$ which is also the superior anterior limit of the SLS. The length of the ALS measured an average of $26.28 \pm 4.29 \mathrm{~mm}$. The SLS continues posteriorly until the posterior insular point (PIP) ${ }^{39}$ where it ends and meets the ILS. The SLS measured an average length of $51.73 \pm 6.50$ $\mathrm{mm}$ (Table 1).

Finally, the ILS extends from the PIP to the meeting point of this sulcus with the limen insula, a point that we refer to as the "temporal limen point" (TLP). The ALS and SLS are straight sulci, but the ILS is curved and can be subdivided into an anterior and a posterior part. The anterior part of the ILS is medial to the polar planum of the temporal lobe and has a more horizontal disposition, whereas its posterior part is medial to the temporal planum and assumes a more vertical disposition. A point placed at the transition between the anterior and posterior parts of the ILS, referred to as the "posteroinferior insular point," lies medial to the anterior part of Heschl's gyrus and corresponds to the lateral projection of the lateral geniculate body (LGB) of the thalamus. ${ }^{42}$ The average total length of the ILS in our study was $44.02 \pm 4.52 \mathrm{~mm}$, with its anterior part (from TLP to LGB) measuring $29.22 \pm$ $4.04 \mathrm{~mm}$ on average (Table 1).

Special attention was directed to the limen insula and to the points where it joins the SLS and ILS, referred to here as FLP and TLP, respectively, since this region is promptly and easily seen as the neurosurgeon splits the sylvian fissure and faces the insular surface. The AIP and PIP, already considered in the literature, ${ }^{38,39}$ lie deep under the frontoparietotemporal opercula and can only be seen during surgery after great retraction or substantial resection of the opercula. Most measurements were made from the limen, FLP, and TLP in an attempt to make them more useful during surgery.

A central insular sulcus is found at the insular surface, subdividing this surface into an anterior and a posterior portion. It was found to be continuous and running from the limen insula to the SLS in the great majority (95\%) of the specimens. The point where this sulcus meets the SLS was found $11.55 \pm 7.23 \mathrm{~mm}$ anterior to the PIP.

The anterior portion of the insula is composed of small insular gyri, which usually converge to form the insular apex. Eleven specimens (58\%) had 3 short gyri in the anterior portion of the insula, 7 (37\%) had 4 short gyri, and just $1(5 \%)$ had 2 short gyri. The insular apex was well defined in only 11 cases (68\%), and because of this lack of consistency, the limen insula was considered a more reliable landmark to be used in surgery. A transverse gyrus was present in all cases, connecting the bottom of the insula anteriorly to the posterior orbital gyrus, and an accessory gyrus was seen superior to it, running toward the ALS from the anterior portion of the insula to the orbitofrontal operculum, in 8 specimens (42\%). The posterior portion of the insula is smaller and had 2 long insular gyri in the majority of cases (18 [95\%] of 19 specimens) (Table 1).

\section{Deep Structures}

The removal of the cortical layer that covers the insular surface exposes the extreme capsule and, deeper, the claustrum, which is a thin collection of gray matter located between the extreme and external capsules and which can 
be subdivided into ventral and dorsal portions (Fig. 2). The ventral claustrum lies deep to the anteroinferior region of the insular surface; its gray matter is organized in a loose pattern of islands embedded together with the white fibers of the extreme and external capsules, giving them a more brownish appearance. It also continues inferiorly under the ILS, and although difficult to follow, its continuation seems to merge into the amygdala. The dorsal claustrum is positioned superiorly and posteriorly, and its gray matter is organized in a more compact thin sheet.

The putamen and the globus pallidus, grouped together as the lentiform nucleus, are located deeper between the external and internal capsules (Fig. 2). The putamen is much bigger and has a darker color, and its lateral surface lies underneath most of the insular surface. The globus pallidus is medial to the putamen, and its identification is favored by its pale appearance and its harder consistency, as well as by its posterior (and superior) position in relation to the anterior commissure. The putamen is separated from the globus pallidus by an external medullary lamina, and deeper, the globus pallidus is subdivided by an internal medullary lamina into an external and an internal portion. The lentiform nucleus is entirely contained inside the margins of the central core and was not found under the depths of the insular limiting sulci.

The internal capsule is found medial to the lentiform nucleus and separates the central core into 2 compartments: 1 lateral, already detailed above, and 1 medial, represented by the caudate nucleus and the thalamus. The caudate nucleus encircles the thalamus, and has a head, a body, and a tail. The head of the caudate nucleus is an oval structure located anterior to the thalamus and represents the medial-anterior aspect of the central core. The foramen of Monro marks the transition between the head and the body of the caudate nucleus. As the body of the caudate nucleus encircles the thalamus, it diminishes into a tail and assumes a more lateral position, leaving the thalamus as the medial-posterior aspect of the central core. The tail of the caudate nucleus becomes progressively smaller and continues along the roof of the temporal horn toward the amygdala at the tip of this horn.

The thalamus, considered as part of the diencephalon, is an ovoid structure located at the top of the brainstem and has 4 surfaces. The lateral part of its superior surface forms the floor of the body of the lateral ventricle, with the caudate nucleus and the stria terminalis running along this border, and the medial part of its superior surface is covered by the tela choroidea of the third ventricle and the fornix. The medial surface itself forms the lateral wall of the third ventricle. The lateral surface directly faces the posterior limb of the internal capsule. The posterior surface, also known as the pulvinar, is subdivided by the choroidal fissure into a medial portion, exposed at the quadrigeminal cistern, and a lateral ventricular portion, which forms the anterior wall of the atrium. The medial and lateral geniculate bodies, 2 prominences found in the inferoposterior aspect of the thalamus, mark the inferior limit of the central core. While the medial geniculate body always lies at the top of the lateral mesencephalic sulcus, the lateral geniculate body (LGB) is more superior and anterior and usually flatter than the former.

\section{White Matter Fibers}

The central core is connected to the rest of the brain by an extensive set of white matter pathways (Fig. 2). Short association fibers, connecting adjacent sulci, can be seen immediately under the cortical surface of the brain. As the dissection is carried out progressively deeper, longer association fibers are exposed, interconnecting different lobes and, later, projecting fibers connecting the cerebral cortex to the basal ganglia, thalamus, and brainstem. The commissural system, which connects regions from both hemispheres, is also represented inside the central core by the anterior commissure. The cortical terminations of these fibers are spread over a large area, but they become closer together and form bundles as they move deeper, where they can be more easily identified. Also, as already mentioned, all these fibers funnel to pass through the narrow space between the insular limiting sulci and the lateral ventricle on their way to reach the other cerebral lobes, assuming a shape of a single condensed group of fibers radiating from the central core, called the cerebral isthmus., ${ }^{4,30}$ Inside the central core, these white matter pathways can be grouped into capsules, which are present in different planes of depth under the insular surface.

The extreme capsule is the most lateral and superficial of all capsules, and lies between the insular surface and the claustrum. It is mainly composed of the short association fibers found directly underneath the insular cortex, interconnecting the insular gyri, and continues spreading under the insular limiting sulci to the opercula, linking the insular and opercular cortices.

The external capsule is located at an intermediate depth, between the claustrum and the putamen. It is formed by 3 fiber bundles, all at the same depth, but arranged in a sequential anterior to posterior disposition: the uncinate fascicle (UF), the inferior fronto-occipital fascicle (IFOF), and the claustrocortical fibers (Fig. 2).

The UF is found directly posterior to the limen insula, assuming its same hook-like shape. From this anteroinferior part of the insula, it continues anteriorly to the posterior orbital region, passing under the inferior portion of the ALS, and to the temporal pole, passing under the most anterior part of the ILS.

The IFOF is located directly posterior to the UF, and its fibers continue superiorly, under the ALS and the most anterior part of the SLS, to reach the middle and superior frontal gyri, and inferiorly, under the ILS, to reach the posterior temporal, parietal, and occipital regions. The claustrocortical fibers emerge from the claustrum in a radial pattern, more clearly identified arising from its dorsal portion, and pass under the SLS and the most posterior part of the ILS toward the brain convexity.

The sequential disposition of these fibers was also found under the insular limiting sulci. Under the ALS, the UF was always present, from its beginning at the FLP until a transition point where the IFOF starts. Since there is no natural demarcation between both fiber bundles, we considered this point to be where the fibers became perpendicular to the ALS; the fibers inferior to it continue to the posterior orbital region and belong to the UF, while the fibers superior to it spread superiorly to the frontal lobe and belong to the IFOF. The UF/IFOF transition point at 

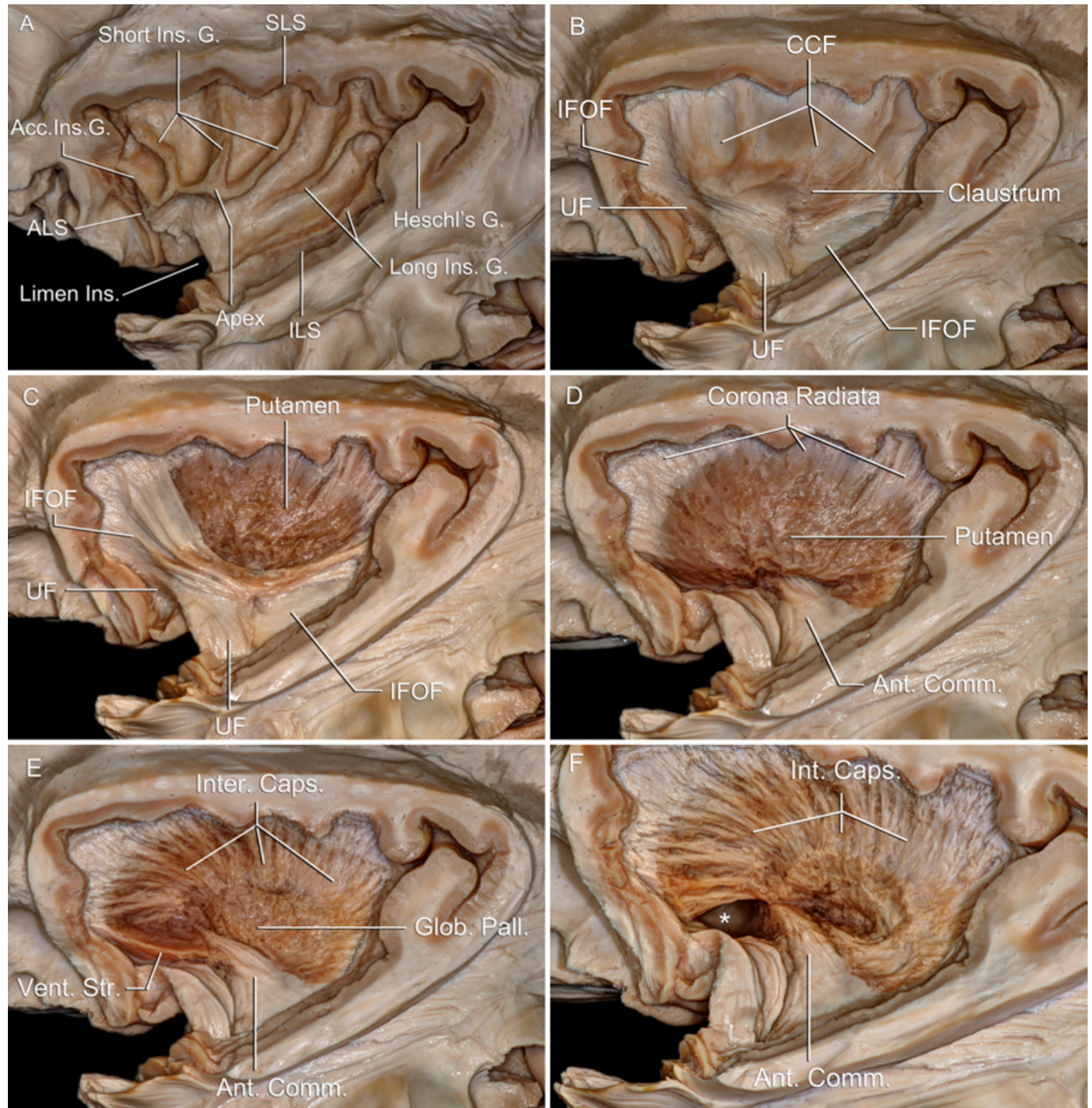

FIG. 2. Lateral fiber dissection of the central core. A: The operculum over the insular surface was removed in this lateral view. The insula was also decorticated; the cortical gray matter of its surface was removed, revealing the thin extreme capsule underneath composed of short association fibers that interconnect adjacent insular gyri and the operculum. Note that the short and accessory insular gyri, at the anterior insular portion, will merge into the insular apex, and the long insular gyri will not reach this apex. B: The dissection is carried more deeply, removing the insular gyri and the extreme capsule. The claustrum is exposed and some major fiber tracts can be distinguished: the uncinate and inferior fronto-occipital fascicles, passing through the ventral claustrum, and the claustrocortical fibers arising from the dorsal claustrum. C: The claustrocortical fibers arise from the dorsal claustrum, and the removal of the former will lead to the removal of the latter, exposing the putamen underneath. Note that the uncinate and inferior fronto-occipital fascicles funnel together below the insular apex, a landmark that can be used to locate them under the insular surface. D: The uncinate and inferior fronto-occipital fascicles were removed and the lateral extension of the anterior commissure is seen. E: The putamen was removed and the globus pallidus is found at its medial and inferior aspect. The anterior commissure crosses the interhemispheric fissure, and as it progresses laterally, it passes at the inferior pole of the globus pallidus through Gratiollet's canal and later underneath the ILS to reach the temporal lobe. The ventral striatum lies anterior and inferior to the anterior commissure. F: The globus pallidus was removed, exposing the entire internal capsule. Fibers from the internal capsule leave the central core as they continue to the cerebral lobes outside the outer limit of the putamen, becoming part of the corona radiata. The internal capsule is not present at the ventral striatum region (white star), making this region a continuous horizontal plate of loose gray matter that extends from lateral to medial along the basal forebrain until the septal area. Acc. Ins. G. = accessory insular gyri; Ant. Comm. = anterior commissure; CCF = claustrocortical fibers; Glob. Pall. = globus pallidus; IFOF = inferior fronto-occipital fascicle; Int. Caps. = internal capsule; Inter. = internal; Long. Ins. G. = long insular gyri; Short Ins. G. = short insular gyri; UF = uncinate fascicle; Vent. Str. = ventral striatum. Anatomical dissections performed by Eduardo Carvalhal Ribas, $\mathrm{MD}$, at Dr. Rhoton's laboratory. Reproduced with permission from the Rhoton Collection (http://rhoton.ineurodb.org), CC BY-NCSA 4.0 (http://creativecommons.org/licenses/by-nc-sa/4.0). 
TABLE 2. Subcortical analysis of all specimens, noting the position of the major fiber pathways deep to the insular limiting sulci

\begin{tabular}{|c|c|c|c|c|}
\hline Description & Mean & Min & Max & SD \\
\hline \multicolumn{5}{|l|}{ Measurements at the ALS } \\
\hline From FLP to UF anterior limit & 0.00 & 0.00 & 0.00 & 0.00 \\
\hline $\begin{array}{l}\text { From FLP to IFOF anterior limit/UF } \\
\text { posterior limit }\end{array}$ & 11.32 & 7.72 & 15.04 & 2.53 \\
\hline \multicolumn{5}{|l|}{ Measurements at the SLS } \\
\hline $\begin{array}{l}\text { From AIP to IFOF posterior limit/ } \\
\text { CCF anterior limit }\end{array}$ & 9.76 & 4.89 & 16.05 & 3.22 \\
\hline $\begin{array}{l}\text { From AIP to superior aspect of the } \\
\text { thalamus }\end{array}$ & 36.50 & 23.76 & 45.89 & 7.36 \\
\hline \multicolumn{5}{|l|}{ Measurements at the ILS } \\
\hline From TLP to UF anterior limit & 0.00 & 0.00 & 0.00 & 0.00 \\
\hline $\begin{array}{l}\text { From TLP to UF posterior limit//FOF } \\
\text { anterior limit }\end{array}$ & 9.96 & 6.36 & 14.48 & 2.21 \\
\hline $\begin{array}{l}\text { From TLP to IFOF posterior limit/ } \\
\text { CCF anterior limit }\end{array}$ & 35.50 & 29.21 & 40.00 & 2.69 \\
\hline From TLP to AC anterior limit & 8.37 & 3.15 & 10.23 & 1.83 \\
\hline From TLP to AC posterior limit & 22.03 & 12.09 & 34.56 & 6.79 \\
\hline $\begin{array}{l}\text { From TLP to visual pathways } \\
\text { anterior limit }\end{array}$ & 10.59 & 6.01 & 17.74 & 3.39 \\
\hline $\begin{array}{l}\text { From TLP to visual pathways poste- } \\
\text { rior limit }\end{array}$ & 34.52 & 28.72 & 39.05 & 3.47 \\
\hline From TLP to amygdala anterior limit* & -4.31 & -7.12 & -2.04 & 1.54 \\
\hline From TLP to amygdala posterior limit & 9.25 & 5.45 & 14.36 & 2.55 \\
\hline $\begin{array}{l}\text { From TLP to temporal horn anterior } \\
\text { limit }\end{array}$ & 4.40 & 0.00 & 9.48 & 2.87 \\
\hline
\end{tabular}

$\mathrm{AC}=$ anterior commissure $\mathrm{CCF}=$ claustrocortical fibers.

The distances of the anterior and posterior limits of each fiber pathway are shown in millimeters and were measured from the beginning of the corresponding insular limiting sulcus (FLP for the ALS, TLP for the ILS, and AIP for the SLS).

* Negative values indicate that the anterior limit of the amygdala was found anterior to the reference point (TLP).

the ALS was found at an average of $11.31 \pm 2.52 \mathrm{~mm}$ from the FLP, and all the ALS superior to this point was related to the IFOF (Table 2).

The identification of the claustrocortical fibers was easier because they are attached to the claustrum cells, and the peeling of the former leads to the removal of the later. A transition point between IFOF and claustrocortical fibers is present under the SLS, at an average of $9.76 \pm 3.22$ $\mathrm{mm}$ posterior to the AIP, and all the SLS posterior to this point was related to claustrocortical fibers (Table 2).

Under the ILS, the UF is the anterior-most fiber bundle to be found. Its fibers are present from the beginning of the ILS, at the TLP, until the point of transition with the IFOF. Again, this point is not clear and was positioned where the fibers became perpendicular to the ILS; the fibers anterior to this point assume an anterior course to the temporal lobe and belong to the UF, whereas the fibers posterior to it spread to more posterior regions of the brain and are related to the IFOF. The UF/IFOF transition point at the ILS was seen at an average of $9.95 \pm 2.21 \mathrm{~mm}$ posterior to the TLP. Another transition point between the IFOF and the claustrocortical fibers is found at the ILS, at an average of $35.5 \pm 2.68 \mathrm{~mm}$ posterior to the TLP. As a result, the claustrocortical fibers are related to the posterior-most part of the ILS and the posterior part of the SLS as well (Table 2).

It is interesting to note that the ventral claustrum is organized in a loose pattern of gray matter islands, and fibers from the UF and IFOF are found passing superficially and underneath these islands in this region. Thus, these fibers can be understood to contribute to both the extreme and external capsules at the anteroinferior part of the insula. The fibers that emerge from the dorsal claustrum, and form the claustrocortical fibers, arise from both the lateral and medial aspects of this nucleus and can be seen as belonging to both capsules, as well as the superior and posterior parts of the insula.

The UF and IFOF fibers are spread under the ALS and SLS, but funnel together at the anteroinferior part of the insula, to spread again under the ILS. The apex of the insula, although not evident in all specimens and not having a constant location, was always superior to this UF/IFOF funneling, and can be used as a landmark to locate the fibers under the insular surface.

The removal of the lentiform nucleus exposes the internal capsule, which represents most of the projection system of the brain-most importantly, the pyramidal tract, corticopontocerebellar tracts, and all of the thalamic radiations ${ }^{15}$ (Fig. 2). This thick group of fibers separates the striatum into the putamen laterally and the caudate nucleus medially and is classically divided into an anterior limb, genu, posterior limb, and retrolenticular and sublenticular portions. The internal capsule fans out in a radial orientation to reach the cortex. Because it passes the outer limit of the caudate nucleus, a circular line that also corresponds to the outer limit of the central core, the internal capsule is named the "corona radiata."

The visual radiation is present at the retro- and sublenticular portions of the internal capsule; it originates at the region of the LGB and reaches the occipital mesial cortex, passing underneath the ILS on its course. The fibers that emerge at the inferior aspect of the LGB first describe an anterior trajectory toward the temporal pole and later turn inferiorly and posteriorly to terminate along the inferior lip of the calcarine fissure (lingual gyrus). This curve, which runs lateral to the temporal horn, is called Meyer's loop. The fibers that arise from the posterior aspect of the LGB have a more direct course to the occipital pole, and the ones that emerge from the superior aspect of the LGB progress to the superior lip of the calcarine fissure (cuneal gyrus). Under the ILS, the anterior and posterior limits of visual radiations were found at an average of $10.58 \pm 3.38$ $\mathrm{mm}$ and $34.51 \pm 3.4 \mathrm{~mm}$ posterior to the TLP, respectively (Table 2). The auditory radiation, present at the sublenticular portion of the internal capsule, emerges from the medial geniculate body and projects to the most anterior transverse temporal gyrus, also called Heschl's gyrus.

Lateral to the atrium of the lateral ventricle, and hence already outside the central core, fibers of the IFOF, anterior commissure, and optic radiation all correspond to the so-called sagittal stratum..$^{22}$ These fibers are found above 

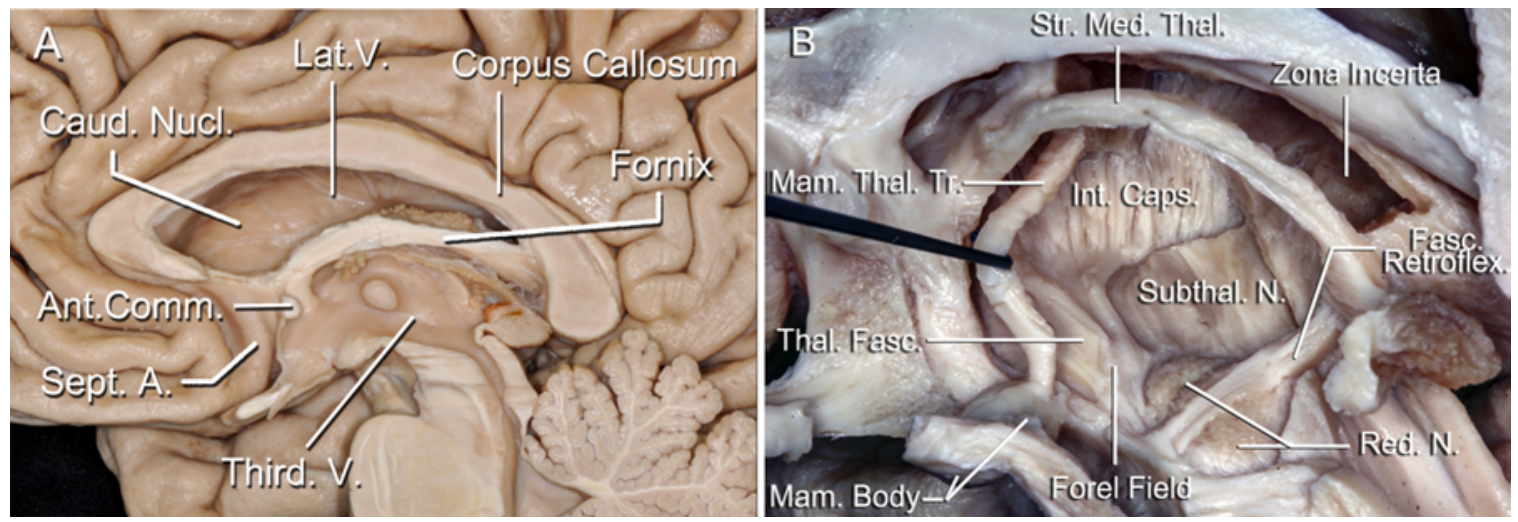

FIG. 3. Medial fiber dissection of the central core. A: Some structures that border the central core can be seen at this medial view of the brain, although the central core is not directly present at the medial surface of the brain. The septal area and the fornix (both considered limbic structures), the third ventricle, and the head and body of the lateral ventricle are medially positioned in relation to the central core. The corpus callosum and the anterior commissure, both composed of commissural fibers that cross the midline and interconnect both cerebral hemispheres, are found around the central core, passing first over it and then later its anteroinferior border. B: The thalamus was removed, and some fiber pathways can be distinguished inside the central core. The habenular nuclei, found anterior and inferior to the pineal gland, receive afferents from limbic structures through the stria medullaris thalami and send efferent outputs to the interpeduncular nucleus through the fasciculus retroflexus. The mammillothalamic tract is found at the anterior aspect of the thalamus, the Forel field and thalamic fascicle are at its basal limit, and the internal capsule is at its lateral border. The subthalamic and red nuclei are inferior to the thalamus. Caud. Nucl. = caudate nucleus; Fasc. Retroflex. = fasciculus retroflexus, Lat. = lateral; Mam. Body = mammillary body; Mam. Thal. Tr. = mammillothalamic tract; N. = nucleus; Sept. A. = septal area; Str. Med. Thal. = stria medullaris thalami; Subthal. = subthalamic; Thal. Fasc. = thalamic fascicle; V. $_{\text {. }}$ ventricle. Anatomical dissections performed by Eduardo Carvalhal Ribas, MD (Panel A) and Kaan Yağmurlu, MD (Panel B), at Dr. Rhoton's laboratory. Reproduced with permission from the Rhoton Collection (http://rhoton.ineurodb.org), CC BY-NC-SA 4.0 (http://creativecommons. org/licenses/by-nc-sa/4.0).

the tapetum fibers, which are callosal (splenial) fibers that run toward the temporal lobe in between the optic radiation and the ependyma. ${ }^{15}$

The anterior commissure connects mostly temporal mesial regions of both hemispheres that are not connected by the corpus callosum. It crosses the interhemispheric fissure perpendicular to it, dividing the columns of the fornix of each side into a major postcommissural component (directed to each mammillary body) and a smaller precommissural component (directed to each septal region). The anterior commissure has a cylindrical appearance along the midline, and as it progresses laterally it divides into a very tiny and involuted ventral extension directed anteriorly toward each olfactory region and a lateral extension directed to the temporal lobe. The lateral extension passes through a tunnel (Gratiollet's canal) at the ventral aspect of the globus pallidus, continues under the putamen and underneath the ILS, and then progressively fans out along the dorsal aspect of the temporal lobe, joining the sagittal stratum as it courses laterally. Under the ILS, the anterior commissure fibers were located deep to the UF/ IFOF plane and superficial to the interior capsule plane, and its anterior and posterior limits were $8.37 \pm 1.83 \mathrm{~mm}$ and $22.02 \pm 6.79 \mathrm{~mm}$ on average posterior to the TLP, respectively (Table 2).

The mammillothalamic tracts represent an important connection between the hypothalamus and the central core (Fig. 3). They are located within the lateral wall of the third ventricle, projecting from each mammillary body to the anterior thalamic nuclei. The stria terminalis is another small bundle of fibers that connects to the limbic system. It originates at the amygdala, continues posteriorly in a groove between the caudate nucleus and the thalamus, and ends under the head of the caudate at the bed nucleus of the stria terminalis. ${ }^{16}$

The thalamus is divided by an internal medullary lamina into a medial portion, which harbors the medial and dorsal thalamic nuclei, and a lateral portion, related to the lateral and ventral nuclei. This lamina also bifurcates anteriorly and separates the anterior thalamic nucleus from the rest of the thalamus. An external medullary lamina covers the lateral and dorsal aspects of the thalamus, and lateral to this lamina lies the thin reticular nucleus of the thalamus.

There is no natural division between the central core and the brainstem, but we established the inferior limit of the central core as the plane where the optic tract encircles the top of the midbrain to reach the LGB. The subthalamic region, described as located between the dorsal thalamus and the hypothalamus, is present underneath this plane and was understood as partially included in the central core (Fig. 3). This region harbors the subthalamic nucleus and the zona incerta, located at the bottom of the reticular nucleus of the thalamus and also known as the thalamic fascicle or field $\mathrm{H} 2$ of Forel. ${ }^{25}$ The globus pallidus is lateral to the internal capsule, and most of its projections terminate at the substantia nigra, in the midbrain. Other projections from the globus pallidus are represented by the lentiform fascicle and ansa lenticularis, the first passing superiorly and the latter passing inferiorly to the subthalamic nucleus, both reaching the zona incerta medially and coming together as the thalamic fascicle. The subthalamic fascicle is a small connection from the globus pallidus directly to the subthalamic nucleus. ${ }^{25}$ 
This inferior plane that limits the central core also transects other white matter pathways that project to and from the brainstem. The pyramidal tract, which has great functional importance for mobility and which comprises corticospinal and corticonuclear pathways, runs from the genu and posterior limb of the internal capsule to the cerebral peduncle at the midbrain, and later to motor nuclei at the brainstem and spinal cord. The corticopontocerebellar pathways arise from all cortical surfaces of the brain and come together as they course toward the cerebral peduncle, as well to constitute the transverse pontine fibers, which project to the cerebellum along the middle cerebellar peduncle. The superior cerebellar peduncle is the main cerebellar output, and most of these fibers run from the dentate nucleus of the cerebellum toward the red nucleus at the midbrain and then the ventral lateral nuclei of the thalamus. ${ }^{25}$ The medial lemniscus is formed by the spinothalamic tracts, carrying sensory information from the spinal cord to the medial and lateral ventral posterior nuclei of the thalamus. The fasciculus retroflexus connects the interpeduncular fossa to the habenular nucleus, and although these regions are not inside the central core per se, its course is at the inferomedial aspect of the central core.

\section{Anterior Perforated Substance Region}

This anteroinferior part of the central core has poorly defined limits, and hence has to be described separately. This region was defined as superior to the APS itself and anterior and inferior to the lateral extension of the anterior commissure. It also continues medially into the septal region without a clear division, already within the medial surface of the brain (Fig. 2).

The APS corresponds then to the floor and anterior wall of this region, and hence to the roof of the sphenoidal compartment of the sylvian fissure. Its name refers to the many minute orifices created by numerous perforating arteries from the internal carotid, anterior choroidal, and anterior and middle cerebral arteries that penetrate its surface to supply several structures of the central core. Its anterior limit is given by the medial and lateral olfactory striae, with the first ending at the midline and the second continuing laterally until the area where the central core connects to the temporal lobe under the limen insulae. Posteriorly, the APS is bounded by the optic tract; medially, it extends above the optic chiasm into the septal region.

Just above the APS, some fiber pathways can be distinguished originating at the anterior temporal region and amygdala. The most superficial bundle of fibers connects the amygdala with the septal nuclei and is known as the diagonal band of Broca. More deeply, fibers that connect the amygdala to the hypothalamus and to the thalamus are found. This latter bundle is mentioned in the literature as the extracapsular inferior thalamic peduncle, since it is not placed between the caudate nucleus and the putamen like the fibers of the internal capsule. ${ }^{26,27}$ These 3 fiber pathways are grouped together as the "ansa peduncularis" and can also be understood as ventral amygdalofugal pathways, as opposed to the stria terminalis, which constitutes a dorsal amygdalofugal pathway. The ansa lenticularis is also identified within the floor of the central core, more posteriorly, and corresponds to a small group of fibers connecting the globus pallidus and the thalamus.

The substantia innominata should be understood as part of the ventral pallidal striatum, ${ }^{16}$ which lies within the APS region, between the APS itself anteriorly and basally, the anterior commissure posteriorly, and the anterior limb of the internal capsule superiorly. Its structures correspond to a horizontal plate of loose gray matter that lies above the ansa peduncularis (amygdalofugal fibers) described above and which extends from lateral to medial blending medially with the septal area.

The fibers of the internal capsule converge from all cortical surfaces toward the central core but are not found anterior and inferior to the anterior commissure. Although different nuclei have been individualized inside the substantia innominata by means of different methods, ${ }^{5}$ the lack of reliable macroscopic subdivisions makes this region better understood as a macroscopic continuous layer of gray matter, from lateral to medial, where all these nuclei are embedded and may blend together.

Inferior and posterior to the APS region, the amygdala extends posteriorly, covering the most anterior aspect of the temporal horn, and merging superiorly with the globus pallidus deeply to the ILS.

\section{Vascularization}

Numerous small branches arise from the internal carotid, anterior choroidal, and anterior and middle cerebral arteries, and most of them penetrate the APS to supply the basal ganglia and the anterior portions of the internal and external capsules (Fig. 4). As these lenticulostriate arteries ascend, they pass both anterior and posterior to the lateral extension of the anterior commissure, forming 2 peduncles. The peduncle anterior to the anterior commissure reaches the putamen and continues medially to the anterior part of the internal capsule, while the peduncle posterior to the anterior commissure supplies the globus pallidus and continues medially to the posterior part of the internal capsule. The caudate nucleus is supplied by the same arteries, which continue medially inside the internal capsule toward this nucleus. The most anterior and medial of these lenticulostriate arteries, which include the artery of Heubner, were found to ascend directly into the head of the caudate nucleus.

The middle cerebral artery, first running under the APS and sending lenticulostriate branches, reaches the limen insula and bends posteriorly over the insular surface (Fig. 4). At the limen, or close to it, the middle cerebral artery bifurcates (or trifurcates) into trunks; the inferior trunk was always located along the ILS in our specimens, while the superior one coursed in a posterosuperior direction toward the SLS. These trunks, as well as their several main branches, send small arteries into the insular surface to supply its cortex. These small penetrating branches did not seem to reach too deep into the insular subcortical white matter, mostly stopping before the claustrum.

The small thalamoperforating arteries that arise from the basilar tip and proximal segments of the posterior cerebral artery pierce the posterior perforated substance, located behind the optic tracts and along the roof of the interpeduncular fossa, and are directed into the thalamus 

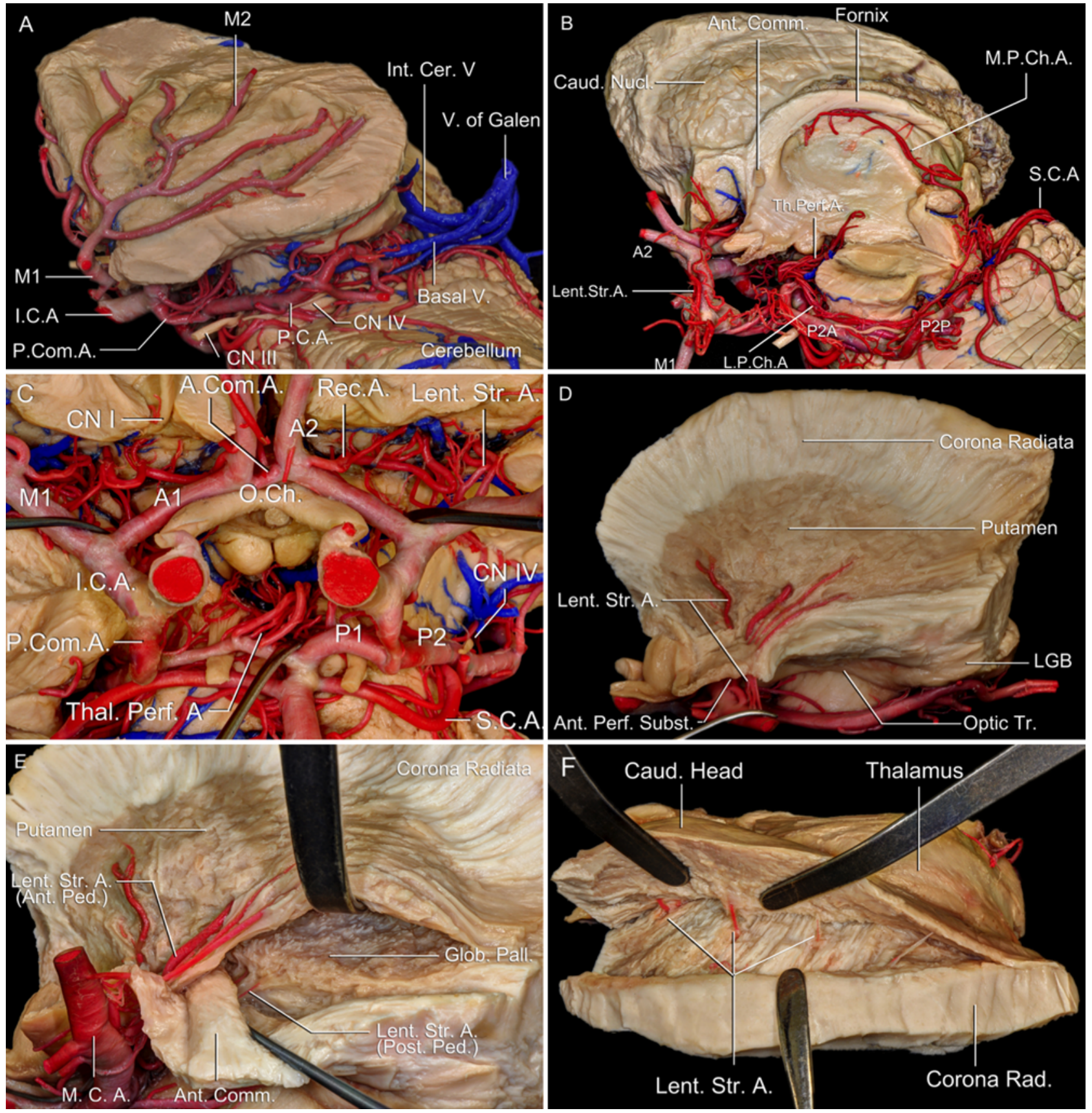

FIG. 4. Arterial supply of the central core. A: All cerebral lobes were removed and the central core is seen on top of the brainstem in a lateral view. The middle cerebral artery (MCA) arises from the internal carotid artery (ICA), and first passes below the central core ( $M_{1}$ segment) and later bends over the insular surface $\left(M_{2}\right.$ segment). Small arterial branches arise from the $M_{2}$ segment of the MCA and supply the most lateral structures of the central core (insular surface, extreme capsule, claustrum, and external capsule). B: The left central core was removed and the arteries related to its vascularization can be seen. The lenticulostriate arteries arise from the $M_{1}$ segment of the MCA, and the thalamoperforating arteries arise from the basilar tip and proximal segments of the posterior cerebral artery (PCA). The lateral and medial posterior choroidal arteries also arise from the proximal segments of the PCA; the first will enter the lateral ventricle, and the second will continue encircling the central core posteriorly and superiorly to supply these regions. C: In this enlarged and inferior view of the central core, note the anterior perforated substance being perforated by several small lenticulostriate arteries arising from the ICA bifurcation and the proximal segments of anterior choroidal artery (ACA) and MCA; meanwhile, the posterior perforated substance is penetrated by the thalamoperforating arteries. D: In this lateral view of the central core, the insular surface, extreme capsule, claustrum, and external capsule were removed. Note the lenticulostriate arteries' trajectory through the anterior perforated substance toward the lentiform nucleus. E: The lenticulostriate arteries form 2 peduncles in relation to the anterior commissure: the anterior peduncle will continue toward the putamen, while the posterior peduncle will continue toward the globus pallidus. FIG. 4. (continued) $\rightarrow$ 
FIG. 4. F: The central core is seen from above, with the caudate nucleus being detached from the internal capsule. The lenticulostriate arteries, after supplying the internal capsule, continue medially toward the caudate nucleus. $A 1=A_{1}$ segment of $A C A ; A 2$ $=\mathrm{A}_{2}$ segment of ACA; Ant. Comm. = anterior commissure; Ant. Ped. = anterior peduncle; Ant. Perf. Subst. = anterior perforated substance; Caud. = caudate; $\mathrm{CN}=$ cranial nerve; Corona Rad. = corona radiata; Int. Cer. V. = internal cerebral vein; Lent. Str. $A .=$ lenticulostriate arteries; L.P.Ch.A. = lateral posterior choroidal artery; $M 1=M_{1}$ segment of $M C A ; M 2=M_{2}$ segment of $M C A$; M.P.Ch.A. = medial posterior choroidal artery; $O . C h .=$ optic chiasm; $P 1=P_{1}$ segment of $P C A ; P 2=P_{2}$ segment of $P C A ; P 2 A=P_{2 A}$ segment of $\mathrm{PCA} ; \mathrm{P} 2 \mathrm{P}=\mathrm{P}_{2 \mathrm{P}}$ segment of PCA; P.Com.A. = posterior communicating artery; Post. Ped. = posterior peduncle; Rec. A. = recurrent artery of Heubner; S.C.A. = superior cerebellar artery; Th. Perf. A. = thalamoperforating arteries; Thal. = thalamo; $\mathrm{Tr}$. = tract; $\mathrm{V} .=$ vein. Anatomical dissections performed by Eduardo Carvalhal Ribas, MD, at Dr. Rhoton's laboratory. Reproduced with permission from the Rhoton Collection (http://rhoton.ineurodb.org), CC BY-NC-SA 4.0 (http://creativecommons.org/licenses/ by-nc-sa/4.0).

and posterior parts of the internal capsule. Other branches emerge from the posterior communicating artery and the posterior cerebral artery as it runs along the lateral aspect of the midbrain; the more anterior branches supply the hypothalamus and the more posterior ones end in the thalamus. The medial posterior choroidal artery encircles the midbrain, ascending posterior to it, and enters the velum interpositum cistern; along its course, it supplies the inferolateral and finally the medial aspects of the thalamus (Fig. 4).

The venous drainage of the central core can be subdivided into a cisternal, a deep, and a ventricular group. The cisternal group is composed of the insular veins that drain the insular cortical surface and are directed to the limen insula, where they join the deep sylvian vein or the deep middle cerebral vein to later empty into the anterior part of the basal vein of Rosenthal (Fig. 5). Occasionally, this group terminates at the sphenoparietal or cavernous sinuses. ${ }^{29}$ Posterior thalamic and epithalamic veins drain the posterior inferolateral and the posteromedial portions of the thalamus, respectively, and empty into the posterior part of the basal vein in its course along the perimesencephalic cisterns.

The deep group is related to the drainage of the central core regions above the anterior and posterior perforated substance regions. The inferior striate veins drain the putamen, caudate nucleus, and internal capsule and converge to exit through the APS, where they join the deep middle cerebral and basal veins. The anteroinferior part of the thalamus is drained by the inferior thalamic veins, which exit through the posterior perforated substance to empty into the posterior communicating or peduncular veins, to also end at the basal vein.

The ventricular group of veins drains the superior and medial aspects of the central core (Fig. 5). These veins course along the medial and lateral walls of the ventricles in a subependymal location and exit these cavities through the choroidal fissure to join the internal cerebral, basal, and great veins. At the frontal horn of the lateral ventricle, anterior thalamic and anterior caudate veins drain the anterior portion the thalamus and the head of the caudate nucleus, respectively; at the body of the ventricle, the thalamostriate, thalamocaudate, and posterior caudate veins drain the body of the caudate nucleus; at the atrium, lateral atrial veins drain the pulvinar of the thalamus and the tail of the caudate nucleus; and at the temporal horn, the inferior aspect of the thalamus and the tail of the caudate nucleus are drained by the inferior ventricular vein located at the roof of this horn.

\section{Subdivision of the Central Core}

Knowing the location of the corticobulbar and corticospinal tracts inside the central core is of great importance, since their lesions usually lead to disabling motor deficits. These tracts arise mostly from the precentral gyrus and project through the genu and the posterior limb of the internal capsule to the cerebral peduncle at the midbrain, progressing to the motor nuclei of the brainstem and spine. The most anterior limit of these fibers was defined by a plane that connects the most anterior aspect of the precentral sulcus to the genu of the internal capsule and to the anterior limit of the cerebral peduncle. This plane can be drawn over the insular surface by studying the lateral projection of the foramen of Monro, because its location is at the same coronal plane as the genu of the internal capsule, and connecting it to the other points mentioned above (Fig. 6). The lateral projection of the foramen of Monro was found at the third short insular gyrus in 10 specimens (71\%), at the fourth in 21 specimens $(21 \%)$, and at the second in 1 specimen (8\%). This vertical line divides the central core into an anterior compartment, related to the anterior limb of the internal capsule, and a posterior compartment, related to the more eloquent parts of the internal capsule. The point that corresponds to the most anterior aspect of the precentral gyrus was found along the SLS at an average of $25.91 \pm 4.48 \mathrm{~mm}$ posterior to the AIP; the point that corresponds to the projection of the anterior aspect of the cerebral peduncle was found along the ILS at $11.73 \pm$ $2.69 \mathrm{~mm}$ (on average) posterior to the TLP (Table 3).

A horizontal plane that includes the lateral projection of the floor of the anterior horn over the insular surface, the foramen of Monro, and the lateral projection of the LGB divides the central core into superior and inferior compartments (Fig. 6). The floor of the anterior horn was laterally projected along the ALS at an average of $11.51 \pm 2.53 \mathrm{~mm}$ superior to the FLP, and the lateral projection of the LBG along the ILS was $29.22 \pm 4.04 \mathrm{~mm}$ (on average) posterior to the TLP (Table 3).

Additionally, the internal capsule and its vascularization coming from its anteroinferior aspect stand inside the central core as a parasagittal plane, and this plane can be used to divide the central core into a lateral and a medial portion. The lateral portion is formed by the insular surface, extreme capsule, claustrum, external capsule, putamen, and globus pallidus, whereas the medial one includes the caudate nucleus and the thalamus. This plane corresponds to a barrier that should not be crossed or damaged during surgeries (Fig. 6).

On the basis of these vertical and horizontal planes, the 

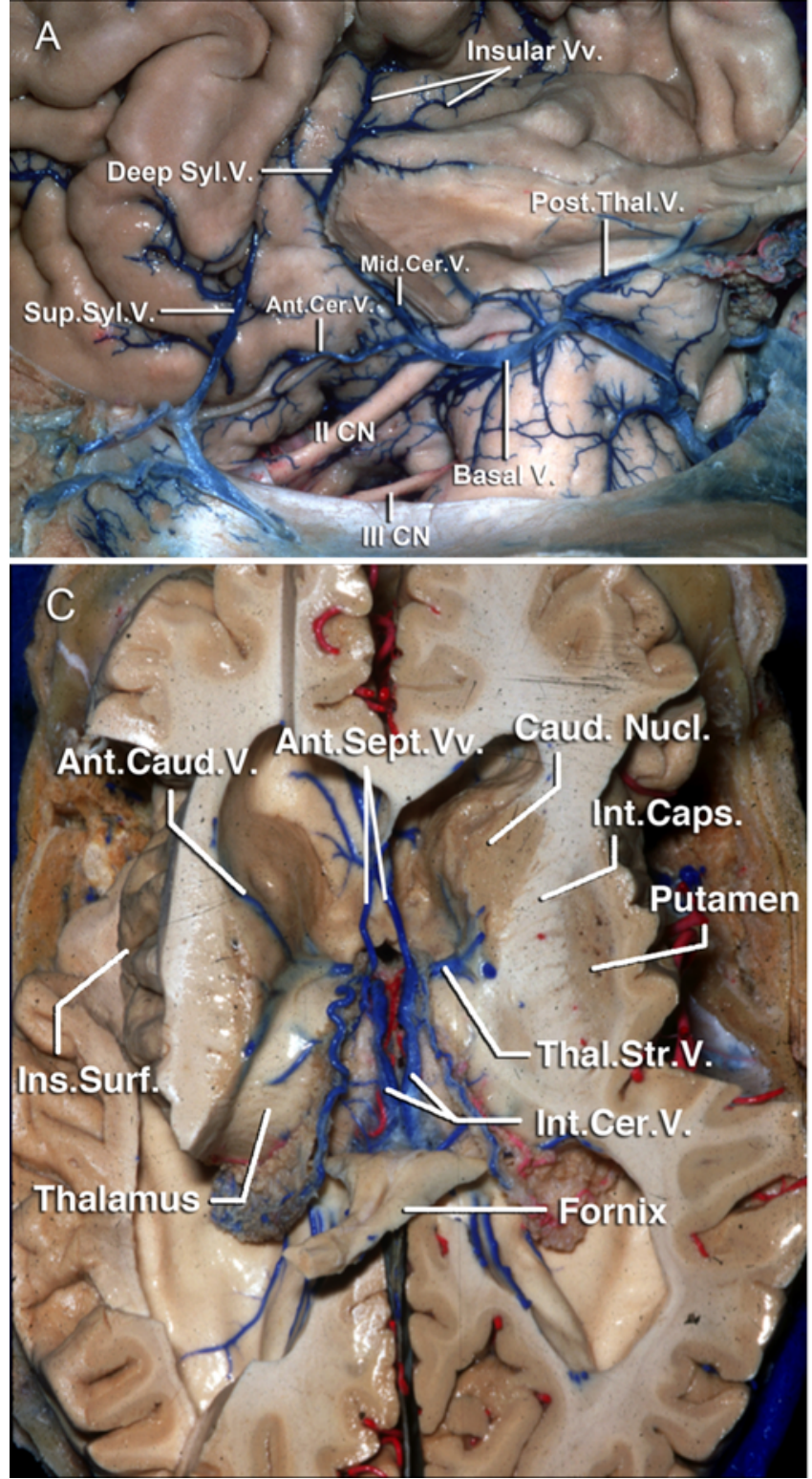

central core can be divided into 4 quadrants, each related to different structures (Figs. 6 and 7).

\section{Anteroinferior Quadrant}

The UF trajectory was located completely inside this quadrant. Also, the anterior perforated substance region and its contents, already detailed, are included here, since the anterior commissure is always slightly anterior and inferior to the foramen of Monro. Because there is no internal capsule inferior to the anterior commissure, very little of the anteroinferior quadrant is related to the internal capsule; just the most inferior portion of the anterior limb can be found at the transition from this quadrant to the superior one. However, most of the vascularization to the internal capsule and basal ganglia runs inside this quadrant, imposing great risk to any attempt to reach deep into it.

Medially, the anteroinferior quadrant continues into the

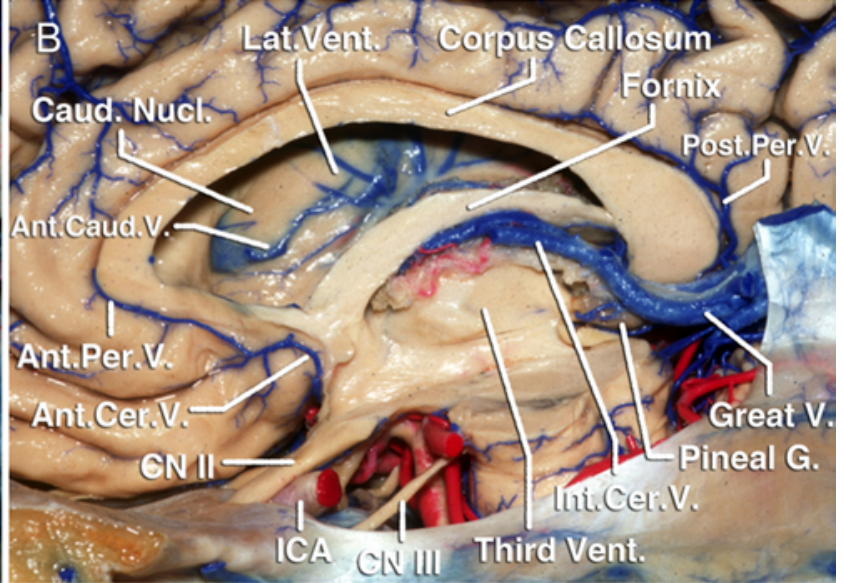

FIG. 5. Venous drainage of the central core. A: The temporal operculum was removed in this lateral view, and the superficial sylvian vein can be seen draining into the sphenoparietal sinuses at the sphenoid bone. The insular veins drain into the deep sylvian vein, which later empties into the anterior part of the basal vein of Rosenthal, and the posterior thalamic vein drains into the posterior part of the basal vein of Rosenthal at its course along the perimesencephalic cisterns. B: The superior and medial aspects of the central core are drained by a ventricular group of veins that pass through the choroidal fissure to join the internal cerebral veins located inside the velum interpositum cistern at the roof of the third ventricle. The internal cerebral veins exit this cistern posteriorly over the pineal gland and are joined by the basal veins to form the great vein. C: In this superior view of an axial cut of the brain, the ventricular veins drain mainly into the thalamostriate veins, which join the anterior septal veins to form the internal cerebral veins. Both fornices were cut and retracted to show the internal cerebral veins at the velum interpositum cistern, located between the third ventricle inferiorly and the fornices superiorly. Ant. = anterior; Caps. = capsule; Cer. = cerebral; G. = Gland; II CN = CN II; III CN = CN III; Ins. = insular; Int. = internal; Mid. = middle; Nucl. = nucleus; Per. = pericallosal; Post. = posterior; Sept. = septal; Sup. = superficial; Surf. = surface, Syl. = sylvian; Thal. $=$ thalamic; Thal. Str. $=$ thalamostriate; Vv. $=$ veins. Reproduced with permission from the Rhoton Collection (http://rho ton.ineurodb.org), CC BY-NC-SA 4.0 (http://creativecommons.org/ licenses/by-nc-sa/4.0).

septal area, meaning that a perpendicular trajectory from its lateral surface will not lead to the opening of the anterior horn.

\section{Anterosuperior Quadrant}

The IFOF fibers are found inside this quadrant and the posteroinferior quadrant. Also, some claustrocortical fibers are present in the anterosuperior quadrant anterior to the vertical plane. Since the vertical plane corresponds to the division between the anterior limb of the internal capsule and its more posterior portions, the depth of this quadrant is not related to the pyramidal tract. Deep to the internal capsule, the head of the caudate nucleus and the frontal horn are found.

\section{Posterosuperior Quadrant}

Most claustrocortical fibers are found inside this quad- 
TABLE 3. Position of the landmarks used for subdivision of the central core

\begin{tabular}{lrrrr}
\hline \multicolumn{1}{c}{ Description } & Mean & Min & Max & SD \\
\hline Landmarks \& distances & & & & 4.49 \\
\hline Precentral sulcus most anterior aspect, distance from AIP at SLS & 25.91 & 17.99 & 34.52 & 2.70 \\
\hline Cerebral peduncle anterior aspect, distance from TLP at ILS & 11.73 & 8.19 & 16.76 & 2.54 \\
\hline Floor of anterior horn, distance from FLP at ALS & 11.52 & 8.06 & 15.75 & 4.04 \\
\hline LGB, distance from TLP at ILS & 29.23 & 22.43 & 37.02 & \\
\hline Distances ${ }^{*}$ from lateral projection of FM to & & & & 3.08 \\
\hline FLP & 21.21 & 14.37 & 24.54 & 3.89 \\
\hline Floor of anterior horn & 23.56 & 15.41 & 29.73 & 2.95 \\
\hline AIP & 27.30 & 20.61 & 31.60 & 2.79 \\
\hline Precentral sulcus most anterior aspect & 15.18 & 11.02 & 20.49 & 4.03 \\
\hline PIP & 30.24 & 24.41 & 38.11 & 2.15 \\
\hline LGB & 19.68 & 16.80 & 24.97 & 2.72 \\
\hline Cerebral peduncle anterior aspect & 14.36 & 10.79 & 19.96 & 2.72 \\
\hline TLP & 18.10 & 11.08 & 21.48 & 3.15 \\
\hline
\end{tabular}

Values are shown in millimeters.

* Measured as length of direct line over the insular surface.

rant, and some IFOF fibers are present here superior to the horizontal plane. The internal capsule found deep to this region includes its genu, its posterior limb, and the retrolenticular and some of the sublenticular portions. Among these fibers, there are optic radiation fibers directed to the superior lip of the calcarine fissure, which have a slightly superior course after arising from the LGB.

Since the horizontal plane connects the foramen of
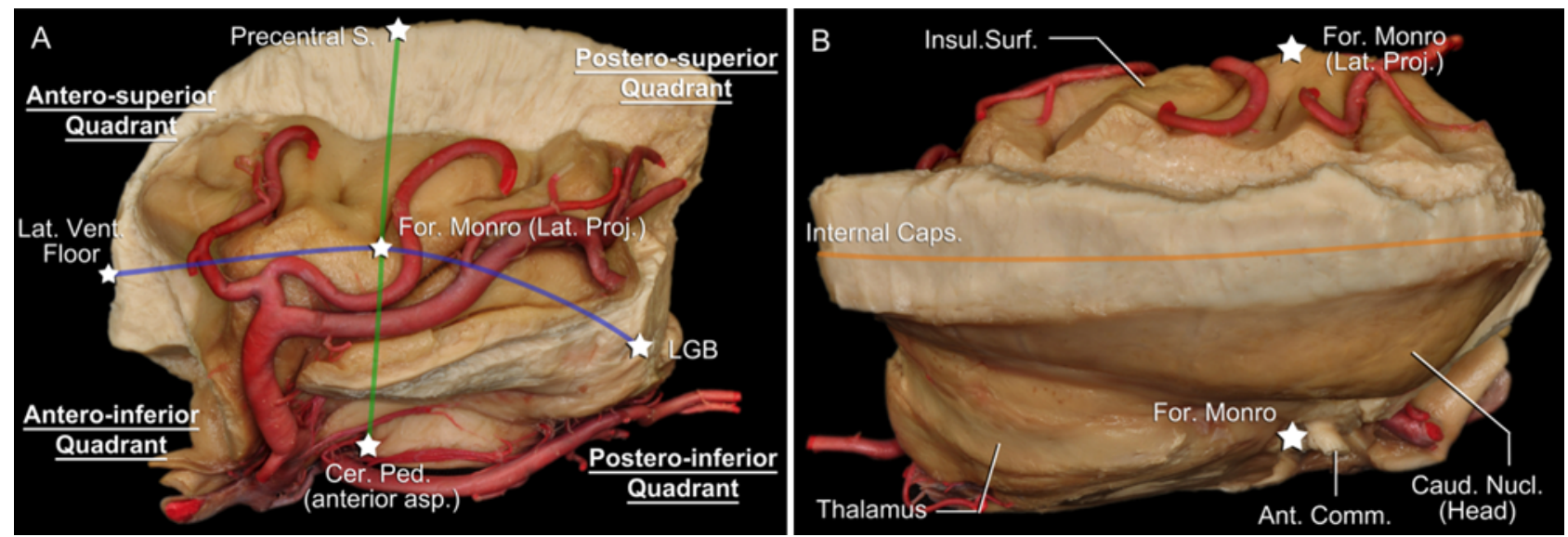

FIG. 6. Central core subdivision. A: In this lateral view, the central core was detached from the other cerebral lobes by a deep cut made along the insular sulci and the limbic structures were removed by opening the choroidal fissure. The central core can be subdivided into quadrants by a vertical line (green) and a horizontal line (blue), both of them meeting at the lateral projection of the foramen of Monro. The vertical line is drawn between the most anterior aspects of the precentral sulcus and the cerebral peduncle, representing the anterior limit of the pyramidal tract, and informs the surgeon that the internal capsule present deep inside the posterior quadrants has the pyramidal tract among its fibers. The horizontal line drawn by connecting the floor of the anterior horn of the lateral ventricle to the lateral geniculate body informs the surgeon that approaches to lesions that are lateral to the anterior horn and body of the lateral ventricle have to be made superiorly to this line, approaches lateral to the third ventricle should be at the posteroinferior quadrant, and approaches to the anteroinferior quadrant will be at the anterior perforated substance region. B: In this superior view of the central core, a line (orange) was drawn along the internal capsule (superiorly) and the lenticulostriate arteries (deep). This line represents a parasagittal plane that should not be crossed during surgeries due to the high functional eloquence of its structures: lesions located laterally should be approached by transinsular lateral approaches, and lesions located medially are better approached by medial routes. Cer. Ped. = cerebral peduncle; For. = foramen; Insul. Surf. = insular surface; Lat. Proj. = lateral projection; Lat. Vent. = lateral ventricle; S. = sulcus. Anatomical dissections performed by Eduardo Carvalhal Ribas, MD, at Dr. Rhoton's laboratory. Reproduced with permission from the Rhoton Collection (http://rhoton.ineurodb. org), CC BY-NC-SA 4.0 (http://creativecommons.org/licenses/by-nc-sa/4.0). 


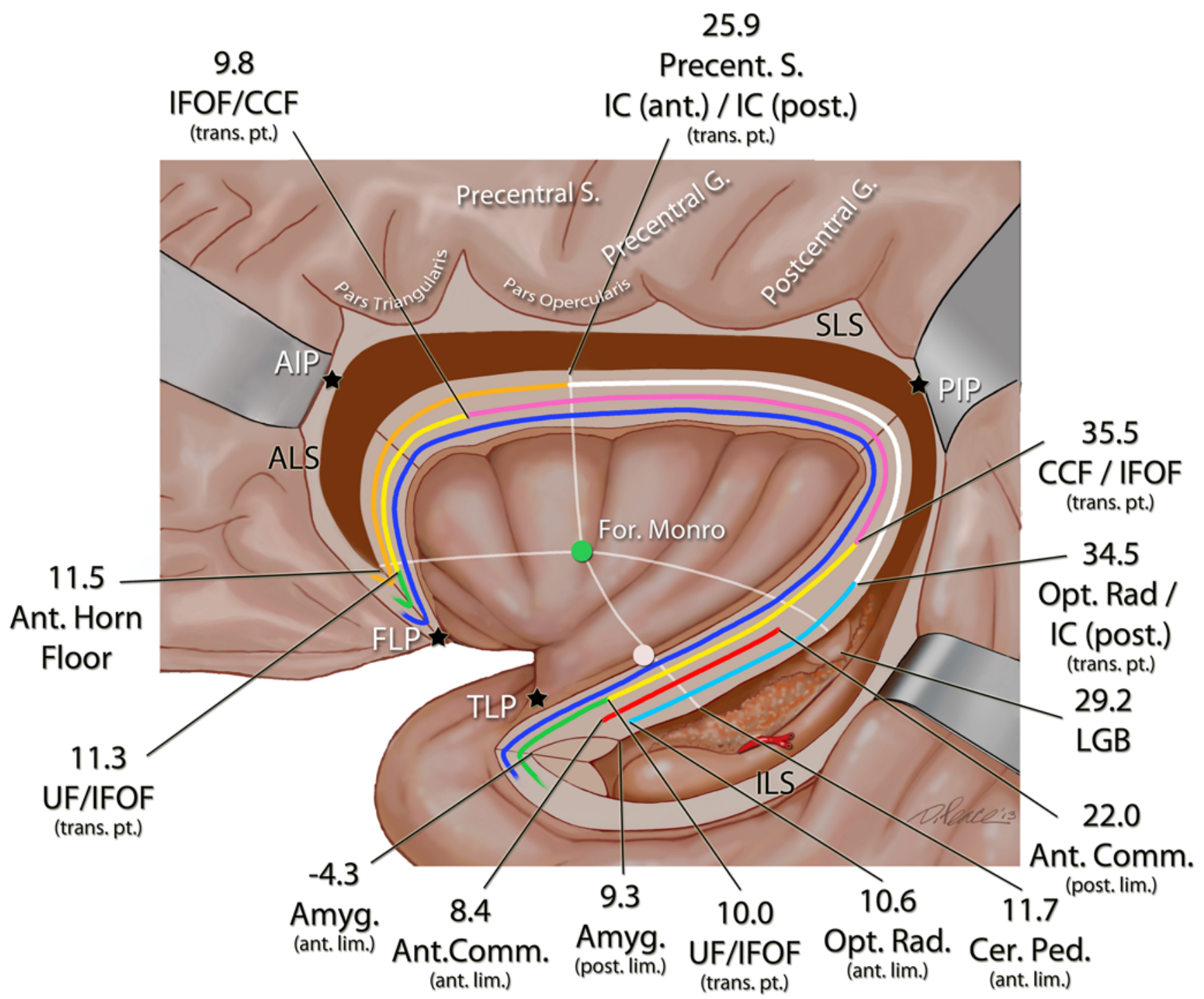

FIG. 7. Artist's illustration showing the position of major fiber pathways underneath the insular limiting sulci. The sylvian fissure was split and the central core is seen in a lateral view. A circular cut was made along the insular limiting sulci, passing through the major fiber pathways of the cerebral isthmus and opening the lateral ventricle. The values shown in the illustration are the mean values (in millimeters) of our measurements of the distances from the anterior and posterior aspects of these major fiber pathways to the reference point of the insular limiting sulci under which they are located; the measurements along the anterior limiting sulcus were made from the frontal limen point, the measurements along the superior limiting sulcus were made from the anterior insular point, and measurements along the inferior limiting sulcus were made from the temporal limen point. The black stars indicate the positions of these points. The positions of the landmarks used for the central core subdivision into quadrants are also reported in Tables 2 and 3. The reference point of the inferior limiting sulcus is the temporal limen point, and the distance measurements posterior to this point were marked as positive values. The anterior limit of the amygdala was found anterior to this reference point, so we used a negative value to indicate that its location was before the reference point (zero). AIP = anterior insular point; ALS = anterior limiting sulcus; Amyg. = amygdala; Ant. Comm. = anterior commissure (red); Ant. Horn Floor = inferior limit of the floor of the anterior horn of the lateral ventricle; ant. lim. = anterior limit; CCF = claustrocortical fibers (pink); Cer. Ped. = cerebral peduncle; FLP = frontal limen point; For. = foramen; IC (ant.) = anterior limb of internal capsule (orange); IC (post.) = internal capsule portions posterior to the anterior limb and to the optic radiation (genu, posterior limb, and part of the retrolenticular portion) (white); IFOF = inferior fronto-occipital fascicle (yellow); ILS = inferior limiting sulcus; LGB = lateral geniculate body; Opt. Rad. = optic radiation (sublenticular and part of retrolenticular portions of internal capsule) (light blue); PIP = posterior insular point; post. lim. = posterior limit; Precent. S. = precentral sulcus; SLS = superior limiting sulcus; TLP = temporal limen point; trans. pt. = transition point; UF = uncinate fascicle (green). Dark blue indicates the extreme capsule. Copyright David Peace (artist). Published with permission. 
Monro to the LGB, the body of the caudate and the thalamus are almost totally included deep inside this quadrant. The body of the lateral ventricle is medial to these structures, and its atrium is posterior.

\section{Posteroinferior Quadrant}

The IFOF fibers and the pyramidal tract, located within the genu and posterior limb of the internal capsule, are located under the insular surface of this quadrant. Also, the fibers of the optic radiation directed to the inferior lip of the calcarine fissure and to the occipital pole are present here. Medial to this quadrant lies the third ventricle.

\section{Discussion}

The tentorium divides the intracranial cavity into an infratentorial compartment, which contains the brainstem and the cerebellum, and a supratentorial compartment, with the 2 cerebral hemispheres divided by the falx and the diencephalic structures. The subdivision of each hemisphere into lobes is based originally on the bones of the skull and has little to do with the functional aspects of each region. The insular lobe, which includes only the insular surface and subcortical white matter, lies deep and is enclosed by the frontoparietotemporal opercula. Although not related to any bone of the skull, the insular lobe has long been recognized as a distinct region since its original description by Reil in 1809.33,39 The concept of a limbic lobe, introduced by Paul Broca in 1877 as including exclusively the cingulate and parahippocampal gyri ${ }^{2}$ and later modified by others to include "medial portions of the frontal, parietal and temporal lobes," the septal region, the perirhinal cortex, the entorhinal cortex, and the various parts of the hippocampal formation, ${ }^{25}$ became official with the recent edition of the Terminologia Anatomica.${ }^{13}$ Finally, the "greater limbic system" is a paralimbic belt, encircling the limbic lobe, also including the caudal orbitofrontal cortex, the anterior insula, and the temporopolar cortex.

From a morphological point of view, the central core stands on top of the brainstem inside this C-shaped ring formed by the limbic lobe; the choroidal fissure is a circular cleft and naturally separates the thalamus from the hippocampal formation (fornix and hippocampus). Its anteroinferior limit, referred to here as the anterior perforated substance (APS) region, does not have clear limits and blends anteriorly into the orbitofrontal cortex, medially into the septal region, and laterally into the anterior temporal cortex, connecting the central core with the greater limbic system.

The central core corresponds to a topographic concept, and it includes parts of different cerebral lobes, which are pertinent only to cortical surfaces and subcortical white matter, and also such deep cerebral hemisphere structures as the basal ganglia and thalamus.

The arterial supply and venous drainage of the cerebral lobes are represented by major vessels over their cortical and ventricular surfaces, which send smaller vessels into the brain parenchyma. It is interesting to note that, in addition to this pattern of vascularization, the central core also has deep and penetrating vessels related to its deep struc- tures that are not found in other parts of the brain. Lenticulostriate arteries and striate veins related to the anterior and deep part of the central core converge at the APS, and thalamoperforating arteries and inferior thalamic veins related to the posterior and deep part of the central core converge at the posterior perforated substance.

The central core can also be distinguished from the rest of the brain in that it harbors different cytoarchitectonic and functional features. The cerebral lobes are made up of isocortex and include the great majority of cortical primary, secondary, and tertiary areas dedicated to sensory and motor functions, and they are responsible for higher cerebral functions, such as language..$^{25}$ The limbic lobe is seen as more particularly related to emotions, memory, and behavioral issues. ${ }^{25,34}$ On the other hand, the insular surface shows a gradual transition between the allocortex and isocortex, called the mesocortex, and is implicated in olfactory, gustatory, viscerosensory, visceromotor, somatosensory, and vestibular functions. ${ }^{25,39}$ The basal ganglia, located inside the central core, are involved in the initiation of movements and the selection of motor strategies to improve the performance of a motor task. ${ }^{1}$

The central core is largely connected by white matter pathways to the rest of the brain via the cerebral isthmus and to the brainstem by its natural inferior continuation, serving as a region that integrates different inputs.

The "temporal stem" 17,18 refers to all white matter fibers of the temporal lobe that converge and pass underneath the ILS toward the central core. The temporal stem can be divided into an anterior and medial component, which resembles an anterior temporal peduncle (formed by the ventral amygdalofugal fibers, UF, anterior aspects of the IFOF and of the anterior commissure, and the superior extension of the amygdala toward the globus pallidus), and a posterior and lateral component, constituted by the fibers that pass underneath the ILS between the tip of the temporal horn and LBG (posterior aspects of IFOF and anterior commissure, and optic radiation fibers)..$^{32,35}$

Based on subcortical mapping by electrical stimulation during awake neurosurgical procedures, Duffau ${ }^{6}$ proposed the existence of a ventral and a dorsal stream associated with language, where the former is related to the UF and the IFOF and the latter to the superior longitudinal fascicle and, most importantly, to its arcuate fasciculus component. The ventral stream is present inside the central core and is associated with the semantic components of language; 9,10 the dorsal stream is outside the central core and is related to phonemic components of language. ${ }^{7}$ The IFOF also seems to play a role in awareness, elaboration of visual information for motor planning, reading, and attention, ${ }^{37}$ and other studies have supposed the importance of the UF in category-specific naming tasks for objects and actions ${ }^{21}$ or famous faces..$^{14}$

The subdivision of the central core into quadrants represents an attempt to better understand this region and to possibly lead to more precise and safer surgeries.

The great majority of the pyramidal fibers arise from the precentral gyrus, run deep inside to converge at the genu and posterior limb of the internal capsule, and continue to the cerebral peduncle. ${ }^{12}$ Thus, a vertical plane positioned at the anterior limit of the pyramidal tract can 
inform the surgeon that posteriorly lies the genu and the posterior limb of the internal capsule, comprising not only the pyramidal tract but also the superior thalamic radiations related to the somatosensory information..$^{15}$

A horizontal line drawn over the insular surface along the lateral projection of the floor of the lateral ventricle and the inferior margin of the thalamus can inform the surgeon how high he or she must enter through the insular cortex, if a certain lesion is lateral to the lateral ventricle or to the thalamus, or how close the approach must be to the ILS, if the lesion is below the lateral ventricle or lateral to the third ventricle.

Additionally, the high functional importance of the internal capsule imposes it and its vascularization as a surgical barrier, leading us to divide the central core into a medial and a lateral portion by a parasagittal plane. Medial operative approaches may be the best to reach the caudate nucleus, thalamus, and medial anterior perforated substance region, whereas transinsular approaches are more suitable for lateral structures and lesions.

The anteroinferior quadrant, including the APS region, can be approached by an anterior transinsular route for lesions lateral to the lenticulostriate arteries. Although the UF will most likely be damaged, surgical data suggest that it has an indirect role in the ventral semantic network and can be partially removed without leading to permanent deficits. ${ }^{11}$ Lesions medial to the vascular supply of the internal capsule can be addressed inferiorly through a supracarotid triangle formed by the proximal segments of the anterior and middle cerebral arteries at the orbitofrontal surface, a route described as the supracarotid-infrafrontal approach, or superiorly by an anterior transcallosal approach that extends through the floor of the lateral ventricle. ${ }^{3}$

The suggested surgical approaches for the anterosuperior quadrant include the anterior transinsular approach for superficial lesions and the anterior interhemispheric approach for lesions related to the head of the caudate nucleus. ${ }^{28,31}$ A deep transinsular approach at the dominant hemisphere can damage the IFOF and may lead to language deficits. The anterior limb of the internal capsule, carrying anterior thalamic radiations and most of the corticostriatopallidal fibers, is found deeper within this quadrant, and despite having been related to behavioral disorders, its resection superior to the anterior commissure might not lead to an important functional loss. ${ }^{8,23}$

The posterior transinsular approach ${ }^{28}$ is suitable for lateral lesions at the posterior quadrants, but must not go deep to safeguard the posterior limb of the internal capsule. Although not seen in our study, Türe et al ${ }^{40}$ stated that $3 \%-5 \%$ of cortical insular arteries, most located over the posterior surface of the insula, are long and extend as far as the internal capsule. Also, microangiographic analysis ${ }^{20}$ showed that medullary arteries from the opercular and cortical segments of the middle cerebral artery can also supply the internal capsule, indicating that there may be a risk of infarction even when coagulation is limited to superficial arteries. Medial lesions of the posterosuperior quadrant will likely be related to the thalamus and can be addressed by a posterior interhemispheric or transparietal approach directed to the body or atrium of the lateral ventricle. ${ }^{31} \mathrm{Le}-$ sions of the posterior and inferior aspects of the thalamus can be reached by a posterior interhemispheric or supracerebellar-infratentorial route, respectively.,31 The third ventricle, medial to the posteroinferior quadrant, can be reached by an interhemispheric transchoroidal approach. ${ }^{43}$

Tumors, arteriovenous malformations, and cavernous malformations often occur inside or at the boundaries of the central core. Although some authors have operated on these lesions with good outcomes, $3,28,36$ most neurosurgeons consider this region to be a challenge. Sanai et al. $^{36}$ also proposed a different subdivision of the insular surface into quadrants, with the horizontal line set along the sylvian fissure and the vertical line perpendicular to it along the foramen of Monro, with a more straightforward application for insular gliomas, which were mostly located underneath the anterior half of the insula in their series.

\section{Conclusions}

We have provided a detailed description of the cortical and subcortical anatomy of the central core. Special attention was devoted to the position of each white matter pathway and deep gray matter structure, correlating all of them with the insular limiting sulci. Reporting the measurements in relation to the limen insula represents an attempt to make our findings more useful during neurosurgical procedures, since this region is frequently exposed with the opening of the sylvian fissure.

We proposed a subdivision of the central core into quadrants, highlighting their most important components, and discussed potentially related surgical approaches. Although our study stands only on anatomical and not clinical data, we believe that our findings are also applicable for surgeries.

\section{Acknowledgments}

We highlight the honor that it was to work with Prof. Albert Rhoton Jr.; we will be forever grateful for his teachings, kindness, and friendship. We also deeply appreciate the assistance of and contributions to this article from Ms. Robin Barry, Mr. David Peace, and Ms. Jessica Striley.

\section{References}

1. Alexander GE, DeLong MR, Strick PL: Parallel organization of functionally segregated circuits linking basal ganglia and cortex. Annu Rev Neurosci 9:357-381, 1986

2. Broca P: Sur la circonvolution limbique et la scissure limbique. Bull Soc Anthropol 12:646-657, 1877

3. Chang EF, Gabriel RA, Potts MB, Berger MS, Lawton MT: Supratentorial cavernous malformations in eloquent and deep locations: surgical approaches and outcomes. Clinical article. J Neurosurg 114:814-827, 2011

4. Choi CY, Han SR, Yee GT, Lee CH: Central core of the cerebrum. J Neurosurg 114:463-469, 2011

5. de Olmos JS, Heimer L: The concepts of the ventral striatopallidal system and extended amygdala. Ann N Y Acad Sci 877:1-32, 1999

6. Duffau H: The anatomo-functional connectivity of language revisited. New insights provided by electrostimulation and tractography. Neuropsychologia 46:927-934, 2008

7. Duffau H, Capelle L, Sichez N, Denvil D, Lopes M, Sichez JP, et al: Intraoperative mapping of the subcortical language pathways using direct stimulations. An anatomo-functional study. Brain 125:199-214, 2002 
8. Duffau H, Denvil D, Capelle L: Absence of movement disorders after surgical resection of glioma invading the right striatum. J Neurosurg 97:363-369, 2002

9. Duffau H, Gatignol P, Mandonnet E, Capelle L, Taillandier L: Intraoperative subcortical stimulation mapping of language pathways in a consecutive series of 115 patients with Grade II glioma in the left dominant hemisphere. J Neurosurg 109:461-471, 2008

10. Duffau H, Gatignol P, Mandonnet E, Peruzzi P, TzourioMazoyer N, Capelle L: New insights into the anatomofunctional connectivity of the semantic system: a study using cortico-subcortical electrostimulations. Brain 128:797-810, 2005

11. Duffau H, Gatignol P, Moritz-Gasser S, Mandonnet E: Is the left uncinate fasciculus essential for language? A cerebral stimulation study. J Neurol 256:382-389, 2009

12. Ebeling U, Reulen HJ: Subcortical topography and proportions of the pyramidal tract. Acta Neurochir (Wien) 118:164-171, 1992

13. Federative Committee on Anatomical Terminology: Terminologia Anatomica: International Anatomical Terminology. Stuttgart: Thieme, 1998

14. Glosser G, Salvucci AE, Chiaravalloti ND: Naming and recognizing famous faces in temporal lobe epilepsy. Neurology 61:81-86, 2003

15. Gray H, Standring S, Ellis H, Berkovitz BKB: Gray's Anatomy: The Anatomical Basis of Clinical Practice, ed 39. New York: Elsevier Churchill Livingstone, 2005

16. Heimer L: The Human Brain and Spinal Cord: Functional Neuroanatomy and Dissection Guide, ed 2. New York: Springer, 1995

17. Horel JA: The neuroanatomy of amnesia. A critique of the hippocampal memory hypothesis. Brain 101:403-445, 1978

18. Horel JA, Misantone LJ: Visual discrimination impaired by cutting temporal lobe connections. Science 193:336-338, 1976

19. Klingler J: Erleichterung der makroskopischen Praeparation des Gehirns durch den Gefrierprozess. Schweiz Arch Neurol Psychiatr 36:247-256, 1935

20. Kumabe T, Higano S, Takahashi S, Tominaga T: Ischemic complications associated with resection of opercular glioma. J Neurosurg 106:263-269, 2007

21. Lu LH, Crosson B, Nadeau SE, Heilman KM, GonzalezRothi LJ, Raymer A, et al: Category-specific naming deficits for objects and actions: semantic attribute and grammatical role hypotheses. Neuropsychologia 40:1608-1621, 2002

22. Ludwig E, Klingler J: Atlas cerebri humani. Boston: Little, Brown \& Co, 1956

23. Machado A, Haber S, Sears N, Greenberg B, Malone D, Rezai A: Functional topography of the ventral striatum and anterior limb of the internal capsule determined by electrical stimulation of awake patients. Clin Neurophysiol 120:19411948,2009

24. Martino J, da Silva-Freitas R, Caballero H, Marco de Lucas E, García-Porrero JA, Vázquez-Barquero A: Fiber dissection and diffusion tensor imaging tractography study of the temporoparietal fiber intersection area. Neurosurgery 72 (1 Suppl Operative):87-98, 2013

25. Nieuwenhuys R, Voogd J, van Huijzen C: The Human Central Nervous System, ed 4. New York: Springer, 2008

26. Peltier J, Verclytte S, Delmaire C, Pruvo JP, Godefroy O, Le Gars D: Microsurgical anatomy of the temporal stem: clinical relevance and correlations with diffusion tensor imaging fiber tracking. J Neurosurg 112:1033-1038, 2010

27. Peuskens D, van Loon J, Van Calenbergh F, van den Bergh R, Goffin J, Plets C: Anatomy of the anterior temporal lobe and the frontotemporal region demonstrated by fiber dissection. Neurosurgery 55:1174-1184, 2004
28. Potts MB, Chang EF, Young WL, Lawton MT: Transsylviantransinsular approaches to the insula and basal ganglia: operative techniques and results with vascular lesions. Neurosurgery 70:824-834, 2012

29. Rhoton AL Jr: The cerebral veins. Neurosurgery 51 (4 Suppl):S159-S205, 2002

30. Rhoton AL Jr: The cerebrum. Neurosurgery 51 (4 Suppl):S1-S51, 2002

31. Rhoton AL Jr: The lateral and third ventricles. Neurosurgery 51 (4 Suppl):S207-S271, 2002

32. Ribas EC, Yagmurlu K, Wen HT, Rhoton AL Jr: Microsurgical anatomy of the inferior limiting insular sulcus and the temporal stem. J Neurosurg 122:1263-1273, 2015

33. Ribas GC: The cerebral sulci and gyri. Neurosurg Focus 28(2):E2, 2010

34. Ribas GC: [Neuroanatomical basis of behavior: history and recent contributions.] Rev Bras Psiquiatr 29:63-71, 2007 (Portuguese)

35. Ribas GC, de Oliveira E: [The insula and the central core concept.] Arq Neuropsiquiatr 65:92-100, 2007 (Portuguese)

36. Sanai N, Polley MY, Berger MS: Insular glioma resection: assessment of patient morbidity, survival, and tumor progression. J Neurosurg 112:1-9, 2010

37. Sarubbo S, De Benedictis A, Maldonado IL, Basso G, Duffau $\mathrm{H}$ : Frontal terminations for the inferior fronto-occipital fascicle: anatomical dissection, DTI study and functional considerations on a multi-component bundle. Brain Struct Funct 218:21-37, 2013

38. Tanriover N, Rhoton AL Jr, Kawashima M, Ulm AJ, Yasuda A: Microsurgical anatomy of the insula and the sylvian fissure. J Neurosurg 100:891-922, 2004

39. Türe U, Yaşargil DC, Al-Mefty O, Yaşargil MG: Topographic anatomy of the insular region. J Neurosurg 90:720-733, 1999

40. Türe U, Yaşargil MG, Al-Mefty O, Yaşargil DC: Arteries of the insula. J Neurosurg 92:676-687, 2000

41. Türe U, Yaşargil MG, Friedman AH, Al-Mefty O: Fiber dissection technique: lateral aspect of the brain. Neurosurgery 47:417-427, 2000

42. Wang F, Sun T, Li XG, Liu NJ: Diffusion tensor tractography of the temporal stem on the inferior limiting sulcus. J Neurosurg 108:775-781, 2008

43. Wen HT, Rhoton AL Jr, de Oliveira E: Transchoroidal approach to the third ventricle: an anatomic study of the choroidal fissure and its clinical application. Neurosurgery 42:1205-1219, 1998

\section{Disclosures}

This research was funded by the University of Florida Foundation.

\section{Author Contributions}

Conception and design: EC Ribas, GC Ribas, Rhoton. Acquisition of data: EC Ribas, Yağmurlu. Analysis and interpretation of data: EC Ribas, Yağmurlu. Drafting the article: EC Ribas. Critically revising the article: de Oliveira, GC Ribas, Rhoton. Study supervision: Rhoton.

\section{Correspondence}

Eduardo Carvalhal Ribas, Division of Neurosurgery, University of São Paulo Medical School, R. Abílio Soares, 569, Apt. 121, São Paulo, SP 04005-002, Brazil. email: eribas@gmail.com. 